\title{
LA VERTU DES IMAGES. ANALOGIE, PROPORTION ET MÉTAPHORE DANS LA GENÈSE DES SCIENCES SOCIALES AU XVIII ${ }^{\mathrm{e}}$ SIÈCLE
}

\author{
Frédéric LEFEBVRE
}

\begin{abstract}
RÉSUMÉ : Le newtonisme moral, à la mode au milieu du XVIII ${ }^{\mathrm{e}}$ siècle, n'est pas seulement une métaphore: en vertu du principe de l'unité de la nature, il postule dans la société une loi semblable à la loi des distances en physique, voire une possibilité de mesure. La même règle d'analogie (A/B = C/D) sous-tend le Contrat social de Jean-Jacques Rousseau : en prolongement des métaphores classiques de l'horloge, la définition du gouvernement reproduit l'agencement d'une montre, jusque dans les calculs de proportion qui servent aux horlogers à former le rouage. De même, dans la Théorie des sentiments moraux, Adam Smith se sert de la vieille métaphore de l'œil de l'esprit et des notions plus modernes de la perspective optique pour agencer en système l'ensemble des interactions possibles entre deux personnes - hormis l'intérêt - en faisant varier la distance imaginaire entre les protagonistes selon les passions exprimées et leur intensité. Il est important de restituer ces transferts et ces controverses pour comprendre les enjeux fondamentaux de la sociologie au $\mathrm{XIX}^{\mathrm{e}}$ siècle.
\end{abstract}

Mots-Clés : sociologie, métaphore, montre, perspective, newtonisme, Rousseau, Smith.

ABSTRACT : Moral newtonianism, in fashion by the middle of the XVIII century, is not only a metaphor. Based on the oneness of nature, it assumes a law of society equivalent to the law of distance in newtonian physics, perhaps offering the possibility of measurement. This method of inductive analogy $(A / B=C / D)$ underlies Jean-Jacques Rousseau's Social contract : Rousseau uses classical metaphors of clockwork, modeling his notion of government on a watch and including mathematical proportions used by clockmakers to design the parts. Similarly, in his Theory of moral sentiments, Adam Smith uses the old metaphor of the eye of the mind, modeling his social system on the recent notions of perspective : two persons, when they meet, will regulate their passions - of any type except interest - according to an imaginary distance between them, which varies according to the nature of the passion and its level. It is essential to restore these translations and the subsequent debates in order to understand the implications for sociology throughout the XIX ${ }^{\text {th }}$ century.

KEYWORDS : sociology, metaphor, watch, perspective, newtonianism, Rousseau, Smith. 
Zusammenfassung : In der Mitte des 18. Jahrhunderts neigte man dazu, das Newtonsche Gravitationsgesetz auf den Bereich der Ideen und der gesellschaftlichen Verhältnisse auszudehnen. Dieser "moralischen Newtonianismus » wurde damals in einer Weise verspottet, die an die «Affäre Sokal» erinnert. Aber moralische Anziehung ist mehr als eine Metapher. Ausgehend von der Einheit der Natur wird für die Gesellschaft ein Gesetz postuliert, das dem Entfernungsgesetz der Newtonschen Physik entspricht und vielleicht sogar eine Messung ermöglicht. Diese Methode einer induktiven Analogie $(A / B=C / D)$ liegt Jean-Jacques Rousseaus Contrat social zugrunde: Rousseau benutzt die klassische Metapher eines Uhrwerks zum Entwurf seines Regierungsbegriffs, wobei er auf die Proportionsrechnung zurückgreift, die den Uhrmachern dazu dient, ein Räderwerk zu berechnen. In ähnlicher Weise gebraucht Adam Smith in seiner Theory of moral sentiments die alte Metapher des geistigen Auges, wenn er sein soziales System auf den neuesten Begriffen der optischen Perspektive aufbaut: wenn sich zwei Personen begegnen, dann stellen sich ihre Leidenschaften jeder Art (mit Ausnahme des gegenseitigen Interesses) entsprechend einer zwischen ihnen bestehenden imaginären Distanz ein, die in Abhängigkeit von Art und Intensität der Leidenschaften variiert. Man muß diese Wechselwirkungen und die sich daraus ergebenden Diskussionen rekonstruieren, um ihre Auswirkungen auf die Soziologie im 19. Jahrhundert zu verstehen.

StIchwörter : Soziologie, Metapher, Uhr, Perspektive, Newtonianismus, Rousseau, Smith.

RESUMEN : El newtonianismo moral, extensión del principio de atracción universal al dominio de las ideas y relaciones sociales, se puso de moda a mediados del siglo xvIII, siendo ocasionalmente denunciado en un tono comparable, en nuestros días, al del «caso Sokal». Sin embargo, la atracción moral no era solamente una metáfora : en virtud del principio de unidad de la naturaleza, sirvió para enunciar una ley social semejante a la ley de las distancias en física, esto es, la posibilidad de medir. La misma regla de analogía $(A / B=C / D)$ subyace al Contrato social de Jean-Jacques Rousseau : la definición de gobierno reproduce el funcionamiento del reloj, prolongando la metáfora clásica, hasta en los cálculos de proporción de los que se sirve el relojero para armar el engranaje. Del mismo modo, en la Teoría de los sentimientos morales, Adam Smith se sirve de la vieja metáfora del ojo del alma y de nociones ópticas más modernas para organizar sistemáticamente el conjunto de las interacciones posibles entre dos personas - excepto el interés -, haciendo variar la distancia imaginaria entre los protagonistas según las pasiones que manifiesten y su intensidad. Es importante reconstruir estas transferencias y controversias para comprender los problemas fundamentales de la sociología del siglo XIX.

PalabRAS Claves : sociología, metáfora, reloj, perspectiva, newtonianismo, Rousseau, Smith.

Frédéric LefebVRe, né en 1963, a soutenu une thèse de doctorat en sociologie intitulée «La montre et l'univers. Métaphores et modèles de la société (de Rousseau à Durkheim)». Son domaine de recherche est l'histoire de la sociologie ( $\mathrm{XVIII}^{\mathrm{e}}-\mathrm{xx}^{\mathrm{e}}$ siècle).

Adresse : INED, unité « Histoire et populations », 133 bd Davout, F-75980 Paris Cedex 20.

Courrier électronique : lefebvre@ined.fr 
«Il faut avouer, dit Micromégas, que la nature est bien variée. - Oui, dit le Saturnien, la nature est un parterre dont les fleurs... - Ah! dit l'autre, laissez là votre parterre. - Elle est, reprit le secrétaire, comme une assemblée de blondes et de brunes dont les parures... - Et qu'ai-je affaire de vos brunes? dit l'autre. — Elle est donc comme une galerie de peintures dont les traits... - Eh non! dit le voyageur, encore une fois la nature est comme la nature. Pourquoi lui chercher des comparaisons? - Pour vous plaire, répondit le secrétaire. - Je ne veux point qu'on me plaise, répondit le voyageur, je veux qu'on m'instruise. »

Voltaire, Micromégas.

DES IMAGES AUX IDÉES

Une « affaire » peut en cacher une autre. Celle qui m'intéresse ici pourrait s'appeler l' «affaire Listonai ». Elle ne date pas d'aujourd'hui, comme l' «affaire Sokal », ni de 1867, quand James Clerk Maxwell doutait des connaissances scientifiques des philosophes, mais de 1761, époque de l'« esprit philosophique » par excellence, cet esprit d'observation et de justesse « qui rapporte tout à ses véritables principes », comme dit l'Encyclopédie, mais qui veut aussi plaire et se rendre utile, car «il aime extrêmement la société » ${ }^{1}$. Une époque où l'on prend plaisir à parler de bonheur, de passions, de sentiments, de convenance, de ridicule, dans les salons, les clubs, les cafés, les conversations, les lettres, les journaux, les essais, les romans, etc., sans jamais cesser de raisonner, de classer, de mesurer, de calculer, dans des traités, des tableaux, des codes, des systèmes, des dictionnaires, des encyclopédies. Et où l'on ne craint pas de mêler les deux. Ainsi David Hume se définit-il comme un «ambassadeur arrivant du domaine du savoir vers celui de la conversation», bien heureux que «le divorce entre les "doctes" et les hommes de salon », « grand défaut du siè-

1. Pour plus de précisions sur les références citées en notes, se reporter à la liste finale, infra, p. 74-77. Ici, Encyclopédie, article «Philosophe». Dans sa discussion de l' «affaire Sokal », Jacques Bouveresse rappelle cette phrase de Maxwell, in Bouveresse, 1999, p. 55 : «[...] la valeur de la métaphysique est égale à la connaissance mathématique et physique de l'auteur divisée par l'assurance avec laquelle il raisonne à partir du nom des choses. » Dans son dernier livre, Jean Starobinski rapproche directement l' «affaire Sokal » de « l'effet de scientificité » couramment recherché dans les deux décennies qui précèdent la Révolution française, voir StARobinski, 1999, p. 45-46. Rapprochement qui s'applique aussi bien aux décennies du milieu du XVIII' ${ }^{\mathrm{e}}$ siècle. 
cle dernier », soit désormais oublié ${ }^{2}$. On méconnaîtrait les Lumières, dit aujourd'hui Jean-Marie Goulemot, si l'on ne tenait pas compte de cet «étrange public », aussi «sensible à l'esprit de géométrie quant à la démonstration » que «partisan de l'esprit de finesse, profondément mondain pour ce qui relève du style et de la forme ${ }^{3}$. Un public éclairé où tout circule, où l'on parle le même langage, que l'on s'appelle le «Don Quichotte des mathématiques » (le père Louis Bertrand Castel vu par Voltaire) ou le «Newton (du) monde intellectuel» (Montesquieu vu par Charles Bonnet). Un public qui se prend de passion pour la question de la mesure — du temps, de l'espace, de la température, bientôt du territoire, du travail, de la population, mais aussi du bonheur, du plaisir, et finalement de l'âme : après la «psychométrie » rêvée par Christian Wolff, la mesure d'intensité proclamée par Bonnet s'oppose à la prudence de Maupertuis, qui veut fonder «l'estimation des moments heureux ou malheureux » sur « le produit de l'intensité du plaisir ou de la peine par la durée ${ }^{4}$, mais reconnaît qu'il n'existe pas de mesure exacte des intensités. Un public familier, au moins dans les termes, avec le calcul infinitésimal de Leibniz et ses successeurs, avec l'harmonie de Rameau, avec la molécule organique de Buffon, l'échelle des êtres de Bonnet, etc., autant qu'il est familier avec la lanterne magique, la chambre obscure, les anamorphoses, les folies électriques et autres prestiges de foire, sans oublier les spectacles de marionnettes ${ }^{5} \ldots$ Mais aussi un public en proie à des difficultés qui peuvent nous paraître plus élémentaires, presque anachroniques pour l'époque. Par exemple, les nombres négatifs, qui posent encore un grave problème théologique : si les grandeurs négatives sont des quantités « au-dessous du rien », alors il faudrait accepter l'idée qu'il existe un véritable mal métaphysique, qui ne serait pas simplement une absence ou une imperfection du bien! Ainsi Kant sera un des premiers, en 1763, à s'aventurer dans l'écriture mathématique de l'équation des plaisirs et des peines, en considérant le déplaisir comme «la négative »du plaisir (noté $a$ ), « c'est-à-dire - $a »^{6}$. Volonté de séduire et fascination pour les savoirs «achevés, exhaustifs, austères même ${ }^{7} »$, dit encore Goulemot : voilà l'esprit du temps dans la littérature

2. Hume, 1973-1974, vol. I, p. 25-27.

3. Goulemot, 1989, p. 67.

4. Pierre-Louis Moreau de Maupertuis, Essai de philosophie morale, cité in BrunET, 1929, vol. II, p. 438. Sur Christian Wolff, voir GusdoRf, 1973, p. 27-28. Sur l'intensité chez Charles Bonnet et Pierre-Louis Moreau de Maupertuis, voir Ratcliff, 1997, p. 438-439. Plus généralement, sur la question de la mesure au XVIII ${ }^{\mathrm{e}}$ siècle, voir LABUSSIÈRE, 1994.

5. Sur la vogue de la lanterne magique, voir LE MEN, 1995. Sur le répertoire des marionnettes, qui parodie les succès et les modes du moment, voir MAGnin, 1981. Sur le sens concret $\mathrm{du}$ «lien » social, en rapport avec la manipulation des marionnettes, voir Duflo, 1998.

6. Emmanuel Kant. Essai pour introduire en philosophie le concept de grandeurs négatives, in KANT, 1980-1986, vol. I, p. 275-276. Sur la querelle des nombres négatifs, voir LoTY, 1995 , p. $405-407$.

7. Goulemot, 1989, p. 67. 
essayiste considérée ici, qui n'est pas la littérature savante des académies et des correspondances scientifiques.

Dans les années 1750, une mode (parmi beaucoup d'autres) s'est emparée de cet étrange « esprit philosophique » : le newtonisme moral. Le mouvement a commencé en Grande-Bretagne : Jean Théophile Désaguliers, Alexander Pope, David Hume, David Hartley cherchaient, chacun à sa façon, à définir l'attraction morale qui gouverne les sociétés humaines comme l'attraction physique expliquée par Newton semble fonder l'harmonie de la nature. Bientôt, la France n'est pas en reste. Plus récemment conquise par les thèses newtoniennes, elle se sépare brutalement et exagérément du cartésianisme : ce ne sont plus des tourbillons que l'on veut voir agir un peu partout, mais de l'attraction, de la gravitation, une loi des distances analogue à celle qui règne dans la nature : Lévesque de Pouilly, qui défendait déjà Newton en France dans les années 1710, fonde son essai de « science des sentiments » sur une loi qu'il veut analogue à la gravitation ${ }^{8}$; Morelly postule une attraction morale qui «lie et enchaîne tous les êtres moraux », une « sorte de gravitation » qui vient s'opposer à cette « espèce d'inertie » que représente notre faiblesse, et «nous met dans l'heureuse nécessité d'être bienfaisants » ${ }^{9}$; Rousseau fait dire à sa Julie quelques énormités qui ne plaisent pas à son précepteur — «Corrigez-moi, mon ami, si j'applique mal vos leçons de physique » -, mais Saint-Preux aurait tort de se plaindre de celle-ci : «Oui, mon ami, nous serons unis malgré notre éloignement [...]. C'est l'union des cœurs qui fait leur véritable félicité; leur attraction ne connaît pas la loi des distances ${ }^{10}$. Il faut comprendre : la loi qui veut que l'attraction entre deux corps (célestes) diminue en fonction du carré de la distance. L'amour, sujet sensible : au contraire de Rousseau, l'Italien Cesare Bonesana Beccaria juge « la puissante attraction qui pousse l'un des sexes vers l'autre [...] semblable en bien des points à la gravitation universelle », puisque, comme elle, elle « diminue avec la distance ${ }^{11}$ ! Mais un débat est-il seulement possible? Il sera d'autant plus difficile que la plupart des lectures newtoniennes de l'ordre social sont en réalité pseudo-newtoniennes, réductrices et infidèles à l'esprit du maître, transformant deux forces orthogonales et différentes par nature (attraction/impulsion) en deux forces opposées et comparables

8. Lévesque de Pouilly, Théorie des sentiments agréables où après avoir indiqué les règles que la Nature suit dans la distribution du plaisir, on établit les principes de la Théologie naturelle et ceux de la Philosophie morale, $1^{\text {re }}$ éd. 1747, cité in MAUZI, 1969, p. 240.

9. Morelly, 1950, p. 166 et 244-245.

10. La Nouvelle Hélö̈se, liv. I, lettre 11 et liv. II, lettre 15, in RoussEAu, 1959-1995, vol. II, p. 55 et 236 .

11. Cesare Bonesana Beccaria, Traité des délits et des peines, $1^{\mathrm{re}}$ éd. 1764, cité in Gusdorf, 1971, p. 208. 
(attraction/répulsion), simple avatar du vieux couple théologique amour/ haine ${ }^{12}$.

Alors que certains, comme Gabriel Bonnot de Mably, se renfrognent déjà devant le succès de l'attraction morale ${ }^{13}$, Listonai (de son vrai nom M. Daniel Jost de Villeneuve) semble voler au secours de la victoire. Le Voyageur philosophe dans un pays inconnu aux habitants de la terre, récit de voyage utopique publié en 1761, contient un chapitre intitulé : «De l'attraction intellectuelle, pour servir de supplément à la philosophie de Newton ». On y lit une déclaration comme celle-ci, tout à fait dans le ton des éloges poétiques de Newton ("God said: "Let Newton be" and all was light », écrivait Pope ${ }^{14}$ !) :

«Les vrais physiciens, parmi nous, n'hésitent plus à admettre la force de l'attraction dans toute la nature: mais ils n'en reconnaissent encore les effets que sur la matière. Voici un sectateur de Newton d'un esprit gai et enjoué, saisi de l'enthousiasme de ses disciples, qui a poussé ses découvertes plus loin que son maitre, en démontrant la réalité de cette force sur les objets intellectuels : il achève ainsi de prouver que l'attraction est un principe universel ${ }^{15}$.»

Mais pour fonder une science morale, il faut énoncer des lois. Listonai s'y applique : «[...] l'attraction [...] opère non seulement sur les corps matériels, en raison directe de la masse et inverse du carré de la distance, mais une vérité non moins importante, c'est qu'elle agit pareillement sur les objets intellectuels, en suivant exactement les mêmes lois. » Attraction véritablement universelle, donc, en ce qu'elle règle aussi les jeux de l'esprit: «les idées s'attirent », «le nom attire vivement le souvenir de la chose, et la chose le nom », « la preuve attire le fait et le fait, la preuve », le tout suivant la fameuse loi des distances. "Et tout tient en équilibre», conclut-il, en prenant à parti tous les philosophes illustres :

«Il serait bien singulier que ce système séduisît quelques esprits : quelle révolution ne causerait-il pas dans la république pensante? que deviendraient alors les sublimes abstractions, les profonds raisonnements, les merveilleuses

12. J'ai proposé le terme de «pseudo-newtonisme », in LefEBvRe, 1998a, p. 435-443, en discutant les positions de Newton et de ses successeurs sur deux questions clés de la cosmologie : l'opposition de deux forces fondamentales; le rôle équilibreur d'un centre de l'univers. Sur le newtonisme moral, dont l'histoire reste à faire, on trouvera des éléments in GouHIER, 1970; Gusdorf, 1971; Schlanger, 1971; Starobinski, 1999; Tuzet, 1988.

13. Gabriel Bonnot de Mably, cité in Morelly, 1950, p. 143 : « Il est bizarre de vouloir comparer l'équilibre physique et l'équilibre moral, ou de penser que leur effet est le même. Les forces et contre forces qui agissent dans le monde de l'homme n'ont pas cette simplicité que l'on constate dans l'univers. Au lieu du système de Newton, il vaudrait mieux les comparer aux tourbillons de Descartes. »

14. Cité in Gusdorf, 1971, p. 165.

15. Listonai est cité in Gounier, 1970, p. 210-211 et Morelly, 1950, p. 142-143. 
conjectures sur l'être en général en tant qu'il est séparé de toute matière? Les fameux Traités de métaphysique d'Aristote, de Descartes, Malebranche, Clarke, Locke, Leibniz, Condillac, etc., rentreraient comme les tourbillons dans le chaos. »

«Comme les tourbillons dans le chaos...»: l'image fait mouche. D'autant plus mouche que tout cela n'était qu'un coup d'ironie, un persiflage avancé selon les règles de l'art! Contre toute attente, dans une note, Listonai dévoile son jeu : ce chapitre n'était qu'une parodie, « une critique enjouée de l'extension que les sectateurs outrés de Newton s'efforcent de donner au principe de l'attraction ${ }^{16} »$ ! Ce n'est donc pas sur Aristote et Descartes que Listonai jette son ridicule, mais sur les petits suiveurs de Newton, tous ceux qui croient effacer les tourbillons de la carte du monde et l'expliquer, au physique comme au moral, par la seule attraction. Voilà une «affaire » bien menée ${ }^{17}$. Une "affaire » stimulante, aussi, qui permet de poser aux sciences sociales en train de naître une question de principe, une question forte: si la relation sociale est spontanément comparée à l'attraction, c'est-à-dire assimilée à une chose de la nature, cette comparaison est-elle seulement une métaphore? N'est-elle pas aussi nécessairement ce qu'on appelle alors une « analogie » (ou « proportion ») ? Et que penser alors de ces expressions d'époque: «lien social», «système social », «rapports entre les hommes ${ }^{18}$, qui nous sont familières aujourd'hui (et pas seulement en sociologie)? Nous ne les connaissons plus que dans un sens vague et figuré, mais est-ce vraiment le cas à l'époque?

En proposant une histoire concrète de l'abstraction dans les sciences sociales, Jean-Claude Perrot a ouvert la voie : Hume, dit-il, « rejette et accepte successivement l'analogie newtonienne. Plus exactement, il en rejette l'usage métaphorique mais il accepte de transporter le même principe explicatif dans le domaine des sciences sociales »; pour sa part, Turgot abandonne ses comparaisons privilégiées, celles de la physique et de l'hydraulique, jusque-là rigoureuses, au profit de métaphores médicales plus lâches, au moment où il ne croit plus à l'économie comme science, où il abandonne son «déterminisme philosophique» pour les notions de «projet », de «politique », de «futur », et s'engage dans l'action gouvernementale ${ }^{19}$. Dans les deux cas, Perrot met l'accent sur ce que, dans un

16. Sur cette forme particulière de moquerie qui se dissimule, voir Bourguinat, 1998.

17. Mais il resterait à étudier la réception du livre, la discussion éventuelle de ses arguments, de son procédé, etc.

18. Les trois expressions se trouvent, p. ex., chez Rousseau : Du contrat social, liv. IV, chap. I, in Rousseau, 1959-1995, vol. III, p. 438; Manuscrit de Genève, liv. I, chap. III, in Rousseau, 1959-1995, vol. III, p. 294; Émile, liv. IV, in Rousseau 1959-1995, vol. IV, p. 493.

19. Perrot, 1992, p. 30, 238 et 252-255. 
autre contexte, Nicole Loraux appelle «la métaphore sans métaphore » : ces « mots cruciaux [...] parce qu'en eux la distinction du propre et du figuré s'abolit ${ }^{20} \gg$. Elle s'abolit, en effet, dans le newtonisme moral de Hume (comme on le verra plus loin), et elle s'abolira encore explicitement chez Émile Durkheim (et certains de ses contemporains : Gabriel Tarde, Ferdinand Tönnies) : Durkheim pose en principe de sa sociologie une « loi de gravitation du monde social» observable dans ses effets, les «forces collectives », expression qu'il nous demande de prendre au sens propre : «Il faut donc prendre les termes à la rigueur [...] »; « Ce n'est pas par métaphore $[. .]$.$» ; «Et, par conséquent, on peut dire sans métaphore$ qu'elles sont des forces ${ }^{21}$ ! Mais, pour de multiples raisons, Durkheim se méprend sur le $\mathrm{XVIII}^{\mathrm{e}}$ siècle, trop dépendant à ses yeux de la mauvaise science cartésienne du XVII ${ }^{e}$ siècle, et n'y reconnaît pas son propre newtonisme moral, en l'occurrence un pseudo-newtonisme comparable à celui de $\mathrm{Kant}^{22}$. On voit que la question introduite par l' «affaire Listonai » ne concerne pas seulement les Lumières et la genèse des sciences sociales : elle touche la nature même des théories sociologiques. Aujourd'hui comme hier, où s'arrête le sens propre, où commence le sens figuré?

Pour aborder cette question, deux ouvrages semblent fondamentaux : l'un de Jean-Jacques Rousseau, l'autre d'Adam Smith, deux auteurs particulièrement intéressants, parce qu'ils réfléchissent à la fois à la genèse des sociétés, à la relation difficile de la morale et de la politesse, à la justesse des métaphores et à la pertinence des mathématiques. Mais aussi parce qu'ils se ressemblent sur un point : tous les deux sont en quelque sorte étrangers dans leur langue. Venus des marges de l'Europe (Genève, l'Écosse), ils ont dû se corriger de leurs idiotismes et conservent de cet apprentissage une acuité particulière aux mots, une grande précision de style $^{23}$. Plus que d'autres, peut-être, ils tiennent leurs métaphores à l'intérieur de leur système, et font de la société une chose proportionnelle à d'autres choses : la montre, dans le Contrat social de Rousseau (1762); la vision perspective, dans la Théorie des sentiments moraux de Smith (1759).

Mais il faut d'abord rappeler le contexte linguistique du XVIII ${ }^{\mathrm{e}}$ siècle, pour délimiter le sens des mots métaphore et analogie dans ce qui va suivre ${ }^{24}$.

20. Loraux, 1999, p. 246.

21. Durkheim, 1967, p. 330 ; Durkheim, 1950, p. 100 ; Durkheim, 1987, p. 348 et 336 ; DURKHEIM, 1925 , p. 36.

22. Sur cette question, voir Lefebvre, 1998a, p. 576-597.

23. Sur Rousseau, voir Meinoz, 1996, p. 95-97. Sur Smith, voir Ross, 1995, p. 89.

24. On ne partira donc pas du sens que ces mots ont aujourd'hui dans les ouvrages sur la question, trop contradictoires dans les termes : Judith Schlanger emploie parfois le mot analogie à la place de métaphore, voir SCHLANGER, 1971, p. 7; Richard Brown fait de l'analogie une catégorie de la métaphore, voir Brown, 1989, p. 173-180: Michel de Coster, qui ne se re- 
Dans son édition de 1718, le Dictionnaire de l'Académie française donne cet exemple : «Homère appelle les Rois Pasteurs des peuples, c'est une belle métaphore. » Par ailleurs, il définit sans ambiguïté l'analogie comme « Terme de mathématique. Rapport, proportion. Il y a même Analogie de deux à trois, que de six à neuf » (ce qui correspond à une égalité de rapports : $\mathrm{A} / \mathrm{B}=\mathrm{C} / \mathrm{D})^{25}$. Le renouvellement de la théorie des langues au milieu du siècle ne viendra pas modifier sensiblement cette distribution des genres $^{26}$. Appartenant à deux registres a priori différents — le langage et la science; les tropes (ou figures) et les systèmes —, la métaphore et l'analogie ne semblent pas être directement confrontées ${ }^{27}$. On ne les rapproche pas quand on spécule sur leur origine. On dit, comme César Du Marsais, que la métaphore est née de ce que «les langues n'ont pas autant de mots que nous avons d'idées », « disette de mots qui a donné lieu à plusieurs métaphores : par exemple, le cœur tendre, le cœur dur, un rayon de miel, les rayons d'une roue, etc. ${ }^{28}$. Tandis que l'analogie aurait son origine dans l'unité supposée de la nature, dans ce que Willem Jacob's Gravesande appelle « l'invariabilité de ces lois qui ne sauraient être sujettes au changement, sans que le genre humain s'en ressente et périsse en peu de temps »: ainsi «l'Auteur de la nature nous a mis dans la nécessité de raisonner par analogie ${ }^{29}$. L'analogie, par extension de sa définition mathématique, est cette méthode d'induction proportionnelle qui permet, en vertu du principe de la raison suffisante, d'étendre nos connaissances à des objets inconnus : comme le rappelle Bonnet, «des effets semblables supposent les mêmes causes $^{30}$ ». Ainsi, de savoir si les planètes sont habitées : «On a beau tourner ces idées en ridicule, dit Rousseau, il est certain que toutes les analogies sont pour cette population, et qu'il n'y a que l'orgueil humain qui soit contre $^{31}$. »

Mais les deux postulats se rejoignent — l'insuffisance de la langue et l'unité de la nature - lorsqu'il est question de définir ce que doit être le langage de la science, le langage de la raison moderne. Dans une conception déjà évolutionniste de l'Histoire, on dit alors que le style métaphorique c'est celui des autres, de ceux qui n'ont pas accès à la raison, celui des civilisations primitives, celui des couches sociales inférieures. Pour William

trouve pas dans les catégories de Brown, fait de la métaphore une catégorie de l'analogie, voir Coster, 1978, p. 20-26.

25. Nouveau dictionnaire de l'Académie françoise, 1994, articles « Métaphore » et « Analogie ».

26. Sur ce contexte, voir Auroux, 1979, p. 60-67 et 278-289, et Tort, 1989, p. 540-542.

27. Du Marsais traite de la métaphore dans son Traité des tropes (1730), Condillac présente l'analogie dans son Traité des systèmes (1749).

28. Cité in Encyclopédie, article « Métaphore ».

29. Cité in CASSIRER, 1966, p. 109-110.

30. Cité in SAvioz, 1948, p. 326.

31. Lettre à Voltaire, in Rousseau, 1959-1995, vol. IV, p. 1067. 
Warburton, « les premiers hommes étant simples, grossiers et plongés dans le sens, ne pouvaient exprimer leurs conceptions imparfaites des idées abstraites [...] qu'à l'aide des images sensibles, qui, au moyen de cette application, devenaient métaphores ${ }^{32} »$. Ou bien c'est le langage des Orientaux que Charles Perrault juge «tout plein de figures, de similitudes, de métaphores, de paraboles et de comparaisons ${ }^{33}$ ». Ou encore, on est «persuadé », comme Du Marsais, «qu'il se fait plus de figures en un jour à la Halle, qu'il ne s'en fait en plusieurs jours d'assemblées académiques ${ }^{34}$ ». L'analogie, au contraire, c'est la fierté des Modernes, « un des flambeaux de la Physique », selon Bonnet ${ }^{35}$. Combiner les observations, généraliser les faits et « les lier ensemble par la force des analogies » : c'est la méthode de Buffon, celle qui permet de « comparer la nature avec elle-même dans ses grandes opérations ${ }^{36}{ }$. C'est le raisonnement qui devrait s'étendre de la science vers les langues, pour les tirer de l'arbitraire et de l'imperfection. Condillac, qui pousse le plus loin la réflexion sur l'analogie, en fait la condition d'une « langue bien faite » (comme l'algèbre, parfaitement analogique), et même le fondement commun à l'invention, au raisonnement et à la parole : «C'est elle qui a fait les Pascal, les Racine et tous les grands écrivains. Ils l'ont aperçue, et ils l'ont prise pour règle : voilà leur génie. L'analogie ne se borne donc pas à faire les langues : elle fait tous les bons esprits ${ }^{37} . »$ On pourrait dire qu'à l'instar de l'analyse des mathématiciens, l'analogie est élevée au rang d'art de penser ${ }^{38}$.

Pourtant, elle n'est pas infaillible: «Quelquefois nous sommes surpris de l'effet imprévu d'une cause, d'où nous nous attendions à voir naître un effet tout opposé », dit encore l'Encyclopédie ${ }^{39}$. Il faut donc l'appliquer avec beaucoup de «circonspection et de sagesse », conseille Bonnet, qui lance l'idée d' " un traité de l'usage et de l'abus de l'analogie [...] qui serait infiniment utile, non seulement dans les sciences physiques, mais encore dans les sciences morales » ${ }^{40}$ ! Dans la même veine, Smith fait la critique de Kepler, qui, «dans chaque science qu'il étudiait, [...] semblait se complaire à trouver quelque analogie entre elle et le système de l'univers »,

32. Cité in Encyclopédie, article « Métaphore».

33. Charles Perrault, dans son Parallèle des Anciens et des Modernes (1688-1692), cité par Starobinski dans son édition de l'Essai sur l'origine des langues de Rousseau, in Rousseau, 1959-1995, vol. V, p. 1543.

34. Cité in Encyclopédie, article « Métaphore ».

35. Cité in Savioz, 1948, p. 326.

36. Buffon, 1986, p. 62-63.

37. Étienne Bonnot, abbé de Condillac, La Langue des calculs, in Condillac, 1947-1951, vol. II, p. 471.

38. Jean Le Rond D'Alembert considère l'analyse mathématique comme un art de penser, étudié aujourd'hui par Éric Brian sous tous ses aspects : matériel, culturel, intellectuel, voir BRIAN, 1994, p. 43-44 et 52.

39. Encyclopédie, article « Analogie ».

40. Cité in SAvioz, 1948, p. 326. 
et, plus généralement, de tous ces « parallèles entre la peinture et la poésie, la poésie et la musique », etc., "systèmes qui ont universellement trouvé leur origine dans les élucubrations de ceux qui connaissaient un art et ignoraient l'autre ${ }^{41}$ ! De l'analogie, on retombe alors dans l'image, l'imagination et l'illusoire ${ }^{42}$. Condillac, à nouveau : "Lorsqu'un peuple choisit mal les analogies, il fait sa langue sans précision et sans goût, parce qu'il défigure ses pensées par des images qui ne leur ressemblent pas, ou qui les avilissent ${ }^{43}$. $\gg$ L'image, considérée soit comme un pur produit des sens, soit comme l'effet des passions originelles ${ }^{44}$, s'oppose aux «idées abstraites », comme chez Turgot, par exemple: "De loin, un chêne ressemble à un orme, et voilà l'idée d'un arbre [...] C'est là l'origine de l'abstraction.» Turgot, parmi d'autres, délimite ainsi une sorte de cadre théorique, où viennent jouer l'image, la métaphore, l'analogie et l'abstraction :

«On voit [les hommes] partir des premières images des objets qui frappent les sens et s'élever par degrés jusqu'aux idées des êtres invisibles et aux abstractions les plus générales. On voit les échelons sur lesquels ils se sont appuyés, les métaphores et les analogies qui les ont aidés, surtout les combinaisons qu'ils ont faites de signes déjà inventés et l'artifice de ce calcul de mots par lequel ils ont formé, composé, analysé toutes sortes d'abstractions inaccessibles aux sens et à l'imagination, précisément comme les nombres exprimés par plusieurs chiffres, sur lesquels cependant le calcul s'exerce avec facilité ${ }^{45}$. »

A priori, la métaphore et l'analogie ne se rencontrent pas, mais a posteriori, dans la maturité de ce que Sylvain Auroux appelle la «sémiotique » des Encyclopédistes, le mot, l'idée et la chose deviennent si liés qu'on doit les considérer dans un rapport ternaire, qu'on ne peut plus les considérer deux par deux ${ }^{46}$. Dans la problématique de l'origine des langues, qui occupe alors une place essentielle, métaphore et analogie ne sont plus nécessairement séparées. Je voudrais suggérer que dans la problématique adjacente de l'origine des sociétés, elle-même indissociable de la question de la nature du lien social, les deux notions se touchent également. De même, dans ce que nous appelons aujourd'hui les images ou métaphores de la société — la société comme organisme, machine, théâtre, jeu, langage,

41. History of astronomy, in SмITH, vol. III, p. 85 et 47.

42. Voir l'association de ces mots chez Rousseau, p. ex., dans l'Essai sur l'origine des langues, chap. III, in Rousseau, 1959-1995, vol. V, p. 382.

43. Cité in Auroux, 1979, p. 284.

44. Rousseau, qui s'oppose à la théorie matérialiste de l'origine des langues, défend cette seconde hypothèse dans l'Essai sur l'origine des langues, chap. II et III, in RoussEAU, 19591995, vol. V.

45. Cité in Perrot, 1992, p. 249-250.

46. Voir Auroux, 1979, p. 66. 
chaos, ordinateur, etc. - , il n'est pas seulement question de mots et d'idées, mais aussi de proportions entre des choses. Même si nous n'employons plus le mot analogie, devenu vague synonyme de ressemblance, et préférons par exemple le mot d'homologie, depuis Ferdinand de Saussure, voire d'homologie structurale - la méthode structuraliste étant par excellence la méthode fondée sur l'égalité de rapports ${ }^{47}$.

\section{LE TEMPS DE LA CITÉ CHEZ ROUSSEAU}

Le Contrat social est un livre difficile, mais certainement pas un livre mystérieux. C'est seulement, dit Rousseau, un de ces « écrits didactiques » dans lesquels il est difficile «d'être clair pour qui ne veut pas être attentif $»^{48}$ ! Nous voilà prévenus : le Contrat social «paraîtra contraire aux idées communes ", car l'auteur y montre "moins d'égards à l'usage qu'à l'analogie quand elle rend le sens plus exact ${ }^{49}$.

Contre l'usage de son siècle, en effet, et contre le sens courant des mots, Rousseau définit le « souverain », le « gouvernement» et l' «État» comme les termes d'une «proportion continue» $(s / g=g / \mathrm{E}$ : le souverain est au gouvernement ce que le gouvernement est à l'État), dont les deux extrêmes sont une seule et même entité considérée sous deux points de vue différents : le «peuple» ou ensemble des «citoyens", «appelé par ses membres État quand il est passif, souverain quand il est actif $»^{50}$. Sous cet appareil mathématique, c'est la théorie de la souveraineté populaire, le modèle républicain inspiré des institutions de Genève, la patrie de Rousseau $^{51}$. Entre les deux, le gouvernement n'est qu'un «intermédiaire», il « reçoit du souverain les ordres qu'il donne » à l'État, il assure leur « communication», leur «mutuelle correspondance», et «sa force n'est que la force publique concentrée en lui ». Rousseau va même plus loin : puisque,

47. Sur les rapports entre analogie et homologie (structurale), voir CosTeR, 1978, p. 47 sq. On peut souligner que Claude Lévi-Strauss, qui a mis l'égalité de rapports au centre de sa méthode de lecture des mythes, a voulu retrouver cette façon de faire chez les auteurs du $\mathrm{XVIII}^{\mathrm{e}}$ siècle, chez Rousseau tout d'abord, chez le musicologue Chabanon par la suite, voir Lévi-Strauss, 1993.

48. Manuscrit de Genève, liv. III, chap. I, in Rousseau, 1959-1995, vol. III, p. 335; Du contrat social, liv. III, chap. I, in Rousseau, 1959-1995, vol. III, p. 395.

49. Du contrat social, liv. II, chap. Iv, in Rousseau, 1959-1995, vol. III, p. 374; Manuscrit de Genève, liv. III, chap. I, in Rousseau, 1959-1995, vol. III, p. 335.

50. Du contrat social, liv. III, chap. I, et liv. I, chap. vI, in Rousseau, 1959-1995, vol. III, p. 396 et 362.

51. Sur l'importance de Genève (et de la Suisse en général) dans la théorie politique de Rousseau, voir Jost, 1961, et Rosenblatt, 1997. Sur la modélisation du problème politique de Genève dans le Contrat social, voir LeFEBvre, 1998b. 
selon la règle de proportion, «il faut, tout compensé, qu'il y ait égalité entre le produit ou la puissance du gouvernement pris en lui-même et le produit ou la puissance des citoyens », prenant «pour exemple le nombre du peuple, comme un rapport plus facile à exprimer », et donnant au mot rapport le sens précis des géomètres, il suggère qu'on peut calculer le nombre optimal des magistrats (on dirait aujourd'hui les fonctionnaires) pour un État donné. Pourtant, arrivé au terme de son application numérique (« la racine carrée du nombre du peuple» pour nombre des magistrats), il semble faire volte-face : «[...] si, pour m'exprimer en moins de paroles, j'emprunte un moment des termes de géométrie, je n'ignore pas, cependant, que la précision géométrique n'a point lieu dans les quantités morales $^{52}$.»

Paul Louis de Bauclair et Guillaume François Berthier, qui sont parmi les premiers à réfuter le Contrat social en France, ne sont pas gênés par cet appareil mathématique, comme nous le sommes souvent aujourd'hui, par anachronisme. Opposés à la république de Rousseau, ils n'ont aucun mal à proposer des proportions plus conformes à l'ordre monarchique, dans lequel « le souverain est bien plus à l'égard du gouvernement que le gouvernement à l'égard du peuple » $(s / g>g / \mathrm{E})^{53}$. Par exemple, cette proportion continue entre le nombre des sujets, des ministres et des lois :

«[...] plus le peuple ou les sujets sont nombreux, plus les lois qui forment le gouvernement seront multipliées; plus le magistrat doit avoir de forces et d'agents pour en opérer ou maintenir l'exécution, et ainsi a contrario. [...] Ainsi, supposant un État composé de 80000 hommes, et dirigé par une somme de 200 lois; vous aurez pour produit en multipliant l'un par l'autre 16000000 ; tirez-en la racine carrée; vous trouverez 4000 pour le corps des ministres du Souverain, ou des membres nécessaires au bon ordre du gouvernement ${ }^{54}$. »

Bien sûr, on parodie l'auteur à abattre. Bauclair écrit : «[...] examinez, fondez, comprenez », puis il ajoute : « [...] considérez, réfléchissez, ordonnez », là où Rousseau avait lancé : «Calculateurs, c'est maintenant votre affaire; comptez, mesurez, comparez ${ }^{55} \gg$ ! Mais la parodie, la satire, l'ironie sont des armes à manier avec talent. Rousseau, bien sûr, n'en manque pas (ses adversaires ne le savent que trop). Avec une ironie qu'on n'a pas assez soulignée, il s'est appliqué pendant plusieurs années à la critique des monarchistes et de leurs métaphores, avant de parvenir à assembler les élé-

52. Du contrat social, liv. III, chap. I, in Rousseau, 1959-1995, vol. III, p. 396-398.

53. BERTHIER, 1988, p. 108.

54. BAUCLAIR, 1981, p. 122-123.

55. Bauclair, 1981, p. 125 et 103 ; Du contrat social, liv. III, chap. IX, in Rousseau, 19591995 , vol. III, p. 420. 
ments de son Contrat social ${ }^{56}$. Déjà dans l'article sur l'« Économie politique », il voulait se dégager de la « comparaison commune et peu exacte à bien des égards » qui associe la société politique à un $\operatorname{corps}^{57}$, argument fort des monarchistes : si le «corps social» est un corps, il ne peut avoir qu'une seule tête, le roi ${ }^{58}$. Dans le Discours sur l'origine et les fondements des inégalités parmi les hommes, il acceptait provisoirement, en attendant d'élaborer sa propre théorie, de suivre «l'opinion commune », qui fait de l'établissement de la société politique un «contrat entre le peuple et les chefs ${ }^{59}$ ». Avec les Écrits sur l'abbé de Saint-Pierre et le Manuscrit de Genève (première version du Contrat social), les attaques se précisent. La «grande famille» de l'État, «ce parallèle fait avec emphase par tant d'auteurs », substitue en vérité «le plaisir de commander» à «l'amour du père pour ses enfants », un amour que n'a certainement pas le chef de l'État monarchique ${ }^{60}$. Le «troupeau », dans lequel Rousseau voyait d'abord une forme «d'association libre » susceptible de figurer les premiers rassemblements humains, devient la métaphore du despotisme, comme dans le Traité sur le gouvernement civil de John Locke (qui rapprochait déjà Homère et La Fontaine, l'Odyssée et Le Loup et l'Agneau ${ }^{61}$ : le despote se fait appeler pâtre (ce sont les «pasteurs d'hommes ») et s'il promet la tranquillité civile, «c'est la tranquillité des compagnons d'Ulysse enfermés dans la caverne du Cyclope, en attendant qu'ils soient dévorés ${ }^{62} »$ ! Enfin, la métaphore du corps est déjà réfutée, en vertu de la thèse centrale du second Discours, celle qui distingue Rousseau de ses contemporains, la thèse de l'homme isolé à l'état naturel :

«La différence de l'art humain à l'ouvrage de la nature se fait sentir dans ses effets, les citoyens ont beau s'appeler membres de l'État, ils ne sauraient s'unir

56. L'ironie et l'humour de Rousseau sont des sujets rarement étudiés, en part. dans le Contrat social. Voir Pintard, 1964.

57. Discours sur l'économie politique, in Rousseau, 1959-1995, vol. III, p. 244.

58. Bauclair, 1981, p. 142.

59. Discours sur l'origine et les fondements de l'inégalité parmi les hommes, II, in RousSEAU, 1959-1995, vol. III, p. 184. Le Contrat social, liv. III, chap. I, malgré l'ambiguîté du titre, achèvera de réfuter l'image du contrat, voir Rousseau, 1959-1995, vol. III, p. 396 : « Ainsi ceux qui prétendent que l'acte par lequel un peuple se soumet à des chefs n'est point un contrat ont grande raison. Ce n'est absolument qu'une commission, un emploi, dans lequel, simples officiers du souverain, ils exercent en son nom le pouvoir dont il les a faits dépositaires, et qu'il peut limiter, modifier et reprendre quand il lui plaît. »

60. Discours sur l'économie politique, in Rousseau, 1959-1995, vol. III, p. 241; Manuscrit de Genève, liv. I, chap. v, in Rousseau, 1959-1995, vol. III, p. 298; Du contrat social, liv. I, chap. II, in Rousseau, 1959-1995, vol. III, p. 352.

61. Discours sur l'origine et les fondements de l'inégalité parmi les hommes, II, in RousSEAU, 1959-1995, vol. III, p. 166. Pour comprendre les enjeux de cette métaphore, voir GoGGI, 1987, et Touchefeu, 1995.

62. Du contrat social, liv. I, chap. II, p. 353 et liv. I, chap. IV, p. 355, in Rousseau, 19591995, vol. III; Écrits sur l'abbé de Saint-Pierre, in RousseAu, 1959-1995, vol. III, p. 609. 
à lui comme de vrais membres le sont au corps; il est impossible de faire que chacun d'eux n'ait pas une existence individuelle et séparée, par laquelle il peut seul suffire à sa propre conservation ${ }^{63}$. »

Rousseau n'aime pas la médecine, encore moins les médecins. Il n'ira jamais loin dans la physiologie pour discuter la métaphore du corps, alors que ses contradicteurs lui opposent un raisonnement qu'ils veulent aussi précis que possible sur les humeurs, le sang, les organes, etc. ${ }^{64}$. Il conserve pourtant la métaphore dans le Contrat social, mais au second plan : plus de circuit sanguin, plus de longue liste d'organes, seulement les fonctions essentielles - la vie, la mort. Des membres, oui, dans « une convention du corps avec chacun » d'eux, mais des membres sans fonction précise, même pour le cerveau et le cœur, rapprochés sans plus d'explication des deux puissances de l'État : l'exécutive et la législative ${ }^{65}$. Pour Rousseau, l'essentiel est déjà ailleurs, dans un critère qui exclut à ses yeux la physiologie : la longévité du pacte social.

«La constitution de l'homme est l'ouvrage de la nature, celle de l'État est l'ouvrage de l'art. Il ne dépend pas des hommes de prolonger leur vie, il dépend d'eux de prolonger celle de l'État aussi loin qu'il est possible, en lui donnant la meilleure constitution qu'il puisse avoir. Le mieux constitué finira, mais plus tard qu'un autre, si nul accident imprévu n'amène sa perte avant le temps ${ }^{66}$. »

«Ouvrage de l'art », « corps artificiel », l'État ne peut être comparé à une famille, à un troupeau ou à un corps vivant, et pas non plus à un levier ou à un char, autres métaphores monarchistes réfutées par Rousseau ${ }^{67}$. Mais il est en tout point comparable à ces machines fragiles que les gestes brusques, les « grands coups d'autorité », comme dit Montesquieu, mettent hors d' « état d'aller » ${ }^{68}$. L'État comparé à une montre : Rousseau a beau clamer son originalité, il emprunte à son tour un lieu commun du siècle,

63. Écrits sur l'abbé de Saint-Pierre, in Rousseau, 1959-1995, vol. III, p. 606.

64. BAUCLAIR, 1981, p. 173.

65. Du contrat social, liv. II, chap. Iv, et liv. III, chap. XI, in Rousseau, 1959-1995, vol. III, p. 374 et 424 .

66. Du contrat social, liv. III, chap. XI, in Rousseau, 1959-1995, vol. III, p. 424.

67. Écrits sur l'abbé de Saint-Pierre, in Rousseau, 1959-1995, vol. III, p. 605. Pour une réfutation ironique du monarque au «levier» de l'État, voir Du contrat social, liv. III, chap. vI, in Rousseau, 1959-1995, vol. III, p. 410 : « Avec un levier suffisant, d'un doigt l'on peut ébranler le monde, mais pour le soutenir, il faut les épaules d'Hercule. Pour peu qu'un État soit grand, le Prince est presque toujours trop petit. » Même ironie contre l'image du «char» ou " attelage», qui sera employée par Bauclair: dans les «vastes Empires», «les rênes de l'État peuvent tomber entre les mains d'un sot, sans que les affaires cessent d'aller »!, voir La Nouvelle Héloïse, liv. VI, lettre 5, in RousSEAu, 1959-1995, vol. II, p. 658.

68. Montesquieu, De l'esprit des lois, XXII, 13, cité et commenté par Hirschman, 1980, p. 69-70; Manuscrit de Genève, liv. I, chap. I, in Rousseau, 1959-1995, vol. III, p. 281. 
après Boisguilbert et Diderot, avant Frédéric II et Herder, en même temps que James Steuart, par exemple, qui donne de la métaphore une version remarquable :

«Il en est des gouvernements comme des machines, plus elles sont simples, plus elles sont solides et durables; plus elles sont composées avec art, plus elles deviennent utiles; mais aussi plus elles risquent d'être hors d'état de marche. La forme spartiate peut être comparée à un coin (wedge), de toutes les puissances mécaniques la plus solide et la plus compacte. Ceux des États modernes peuvent l'être aux montres, qui se dérangent continuellement; tantôt le ressort s'avère trop faible, tantôt trop fort pour la machine : et quand les roues ne sont pas faites en suivant une proportion fixée, par les mains expertes d'un Graham ou d'un Julien Le Roy, elles ne s'accordent (tally) pas bien les unes avec les autres; alors la machine s'arrête, et si on la force, des pièces se cassent; et il faut avoir recours à la main de l'ouvrier pour la réparer ${ }^{69}$. »

De cette version pour ainsi dire canonique de la théorie du « doux commerce », Rousseau se distingue pourtant. Tout d'abord, il masque la métaphore : la montre n'est jamais nommée dans le Contrat social, seulement une «machine». Ensuite, il ne s'attache pas seulement au dérèglement de la montre, mais aussi à sa durée de marche. Comme dit Gérard Namer, Rousseau lit Montesquieu « à partir de Machiavel » - Machiavel qui faisait précisément du temps la «substance du pouvoir», selon l'expression d'Antonio $\mathrm{Negri}^{70}$. Rousseau, qui refuse également l'image de la «balance » chère à Montesquieu, réintègre dans sa machine politique la relation complexe du temps et de la force : «L'on ne peut éviter en politique non plus qu'en mécanique d'agir plus faiblement ou moins vite, et de perdre de la force ou du temps ${ }^{71}$. » Ce qu'il exprime ici, dans un chapitre essentiel (et pourtant négligé) du Manuscrit de Genève, c'est le parallèle qui fondera bientôt le livre III du Contrat social : $1^{\circ}$ la volonté générale du peuple souverain, qui dirige, qui « détermine l'acte », est comme le régulateur (ou échappement, dans la montre à spiral réglant inventée à la fin du $\mathrm{XVII}^{\mathrm{e}}$ siècle); $2^{\mathrm{o}}$ le gouvernement, ministre du souverain et sans autre force que la force publique, est comme le rouage; $3^{\circ}$ l'État (toujours au sens de

69. Steuart, 1967, vol. I, p. 331-332.

70. NAMER, 1979, p. 35; NeGri, 1992, p. 100.

71. Manuscrit de Genève, liv. I, chap. Iv, in Rousseau, 1959-1995, vol. III, p. 296. L'image de la balance, associée de manière conventionnelle à la politique dans l'oraison funèbre du duc d'Orléans (« [...] ces mystères de Cabinet où les destins de l'État sont en secret balancés au poids de l'équité et de la raison »), est implicitement critiquée au moment de l'Émile, car c'est un instrument qui peut être faux, ou volontairement faussé : "Voilà déjà mon petit physicien capable de rectifier des balances avant que d'en avoir vu. » Voir Oraison funèbre de S.A.S. Monseigneur le duc d'Orléans, in Rousseau, 1959-1995, vol. II, p. 1280, et Émile, liv. III, in Rousseau, 1959-1995, vol. IV, p. 442. 
peuple), force commune au tout, est comme le ressort moteur ${ }^{72}$. Et le législateur, bien sûr, est comme le maître horloger de l'époque, qui conçoit la montre et en confie l'exécution à ses ouvriers : «homme extraordinaire dans l'État», il «constitue la république » mais «n'entre point dans sa constitution », il est le «mécanicien qui invente la machine » alors que « d'autres plus sages en régleront les mouvements », viendront la «monte $(\mathrm{r}) »$, la faire « marcher », la « remonter» « quand elle s'arrête ${ }^{73}$.

Nous ne savons pas, et nous ne saurons probablement jamais, comment se présentait la théorie du gouvernement dans le Manuscrit de Genève. Cette partie, Rousseau l'a déchirée un jour. Mais ce qui reste du Manuscrit suffit pour comprendre ce qui manque à Bauclair, à la métaphore du corps, et à Rousseau lui-même jusqu'à l'aboutissement de ses «pénibles recherches » : un troisième terme assurant la communication entre l'âme et le corps, comme la glande pinéale chez Descartes ${ }^{74}$. Ainsi, «l'action de la volonté générale sur la force publique », qui était encore comparée dans le Manuscrit à « l'action de l'âme sur le corps », véritable « abîme de la philosophie », devient dans le Contrat social « l'union de l'âme et du corps », assurée par un «agent propre»: le gouvernement ${ }^{75}$. Mais là où nous comprenons aujourd'hui cette union comme un problème purement physique (ou métaphysique), les contemporains, formés dans Descartes, Leibniz, Mandeville, etc., y mettaient aussi de l'horlogerie : Comenius associait la raison à l'échappement et les désirs aux poids d'une horloge; Descartes mettait en scène deux horloges parfaitement accordées (chose exceptionnelle au moment où il écrit) pour illustrer la perfection divine, qui sait régler ensemble, mais séparément, l'âme et le corps; Mandeville prend l'âme pour «le ressort moteur en nous ». Plus près de Rousseau, Vauvenargues compare la raison à l'aiguille qui indique les heures au cadran d'une montre, et Voltaire traite des passions comme des roues qui font aller les machines de la nature ${ }^{76}$.

72. Du contrat social, liv. III, chap. I, in Rousseau, 1959-1995, vol. III, p. 395-399. Pour une présentation plus détaillée de l'analogie de la montre, qui consiste aussi à rapprocher le problème de la taille des roues du problème de la taille des différents conseils dans une république, voir LEFEBVRe, 1998b.

73. Manuscrit de Genève, liv. II, chap. II, et liv. I, chap. I, in RoussEAu, 1959-1995, vol. III, p. 313 et 281; Lettres écrites de la montagne, lettre IX, in Rousseau, 1959-1995, vol. III, p. 896. Sur la division du travail dans l'horlogerie (on pouvait compter jusqu'à dix-sept métiers différents dans la fabrication d'une montre), voir Encyclopédie, article « Horlogerie ».

74. Manuscrit de Genève, liv. I, chap. Iv, in Rousseau, 1959-1995, vol. III, p. 297; Discours sur les sciences et les arts, part. II, in RoussEAU, 1959-1995, vol. III, p. 18.

75. Manuscrit de Genève, liv. I, chap. Iv, in Rousseau, 1959-1995, vol. III, p. 296; Du contrat social, liv. III, chap. I, in Rousseau, 1959-1995, vol. III, p. 396.

76. Comenius, cité in Mumford, 1973-1974, vol. II, p. 135; sur Descartes, voir Boorstin, 1983, p. 66; MANDEVILLE, 1990, vol. II, p. 141; Vauvenargues, Introduction à la connaissance de l'esprit humain, et Voltaire, Traité de métaphysique, cités in CASSIRER, 1966, p. 129. 
Jusque dans leur précision, les deux métaphores du Contrat social - la société comme montre et la société comme application d'une volonté à une force - se croisent donc déjà avant Rousseau. Mais il appartient à Rousseau de rapprocher la métaphore et l'analogie, en fécondant ces lieux communs par les calculs d'horlogerie, bien connus des contemporains comme exemple de résolution de proportions (ou fractions continues). C'est même un ami de Rousseau, le genevois Jean Romilly, qui en donne le traité dans l'Encyclopédie. Romilly, qui invente en 1757 une montre susceptible de marcher trois cent soixante-dix-huit jours, un record de longévité, un exemple à suivre ${ }^{77}$. Avec la métaphore de la montre, aussi belle - et plus légitime - que celle d'Homère, l' « évidente analogie » qui s'établit entre «le rapport du souverain aux sujets » et le rapport «du gouvernement à l'égard des magistrats » retrouve sa raison d'être ${ }^{78}$. Et, plus généralement, l'appareil mathématique du gouvernement, dont Bauclair réfutera l'« analogie » parce qu'il raisonne lui-même à partir d'une autre métaphore, celle du corps ${ }^{79}$.

\section{L'ESPACE DES RELATIONS CHEZ SMITH}

À l'inverse du Contrat social, la Théorie des sentiments moraux est un succès immédiat : «[...] il est impossible pour un livre aussi sérieux d'être accueilli avec plus de bienveillance ${ }^{80} »$, écrit un témoin depuis Londres. Dans les termes mêmes du jeune Edmund Burke, c'est un excellent travail, aux exemples nombreux et bien choisis, digne d'un homme doué d'un sens peu commun de l'observation, mais aussi un livre au raisonnement puissant, dont les parties se fortifient les unes les autres si naturellement, bref, un des plus beaux édifices de théorie morale qu'on ait jamais $\mathrm{vu}^{81}$.

Sur un sujet aussi «éculé » que celui des «systèmes de morale » (ce sont les expressions de Burke) ${ }^{82}$, Smith parviendrait donc à innover en

77. Voir Jean Romilly, Encyclopédie, article «Frottement». Sur Romilly, citoyen de Genève, maître-horloger à Paris depuis 1752, voir ChapIRo, 1991, p. 132-134.

78. Du contrat social, liv. III, chap. II, in Rousseau, 1959-1995, vol. III, p. 400.

79. Bauclair, 1981, p. 116-118: «On peut douter que l'analogie soit si évidente. Les magistrats ne sont pas au gouvernement ce que les sujets sont au souverain [...] Plus les magistrats sont nombreux, plus le gouvernement est faible; et l'analogie demanderait qu'on dît: Plus les magistrats sont nombreux, plus le gouvernement est fort; car dans la doctrine de l'auteur, plus les sujets sont nombreux, plus le souverain a d'autorité. Et cette dernière proposition doit être le fondement de l'analogie. »

80. Lettre de William Robertson du 14 juin 1759, dans The Correspondence of Adam Smith, in Sмiтн, vol. VI, p. 40.

81. Edmund Burke, cité in David D. Raphael et Alec L. Macfie, introd. à Adam Smith, Theory of moral sentiments, in SмIтн, vol. I, p. 28.

82. Ibid. 
donnant le premier rôle à la sympathie, notion dont il élargit le sens usuel : non plus seulement le sens de pitié et de compassion envers les souffrances des autres, mais «la faculté de partager les passions des autres quelles qu'elles soient ${ }^{83} »$. Ainsi définie, la sympathie devient le principe unique des relations sociales, fonctionnant comme «changement imaginaire de situation ${ }^{84} »$, comme «faculté [...] de nous mettre, par l'imagination », à la place des autres, sans restriction ${ }^{85}$. Dans la configuration de base définie par Smith, un agent exprime un certain sentiment (feeling, passion, sentiment) pour un objet (personne, idée, chose), sous les yeux d'un spectateur. Celui-ci approuve ou désapprouve ce sentiment, selon qu'il lui paraît ou non convenable, c'est-à-dire proportionné à son objet. Mais l'effet naturel de sympathie (l'échange imaginaire des places entre agent et spectateur) peut les conduire, pour parvenir à un meilleur accord (concord), à une meilleure correspondance de leurs sentiments, à modifier, l'un sa passion, l'autre son critère de jugement. James Wodrow, ancien élève de Smith à Glasgow, se souviendra de cette «ingénieuse tentative de rendre compte des principaux phénomènes du monde moral par cet unique principe général, comme la gravitation dans le monde naturel ${ }^{86} »$. Pour ses contemporains, l'ambition de Smith n'apparaît pas moins élevée que celle de Newton.

83. Theory of moral sentiments, I.i.1.5, in SMith, vol. I, p. 10 (trad. Sophie DE Grouchy, marquise de Condorcet, p. 4). On dispose aujourd'hui d'une nouvelle traduction par Michaël Biziou, Claude Gautier et Jean-François Pradeau (1999), qui corrige la plupart des erreurs et omissions de $\mathrm{M}^{\mathrm{me}}$ de Condorcet (1798), en soulignant mieux les métaphores du déplacement et de l'optique. Mais cette nouvelle traduction reste perfectible, car elle atténue trop la construction mathématique de l' «étalon » de convenance des sentiments, les jeux d'échelles d'intensité et de distance, et les notions de «mesure », de «proportion », de « rapport ». De plus, elle sous-estime le registre de la «conversation » et du « ridicule » et surestime la référence au «théâtre », en confondant la position purement spatiale du «spectateur » avec un spectacle sur une scène. Enfin, l'expression «point de vue » n'est pas toujours pertinente pour traduire «in the light of » ou "in the view of », alors même qu'on dispose d'expressions plus courantes à l'époque : «sous le jour de », « au regard de », « aux yeux de ». L'expression « point de vue » conserve, en effet, au XVIII ${ }^{\mathrm{e}}$ siècle un sens proche de son sens technique originel : le point où il faut se placer pour voir correctement un tableau peint selon les règles de la perspective. Dans la logique de Smith, seul le «spectateur impartial » est toujours au « point de vue ». Sur l'histoire de la perspective en général, voir Hamou, éd., 1995, et sur cette question en particulier, voir Frédéric Lefebvre, « De l'art de voir à l'art de vivre. Perspective et point de vue chez Rousseau ", à paraître dans les Études Jean-Jacques Rousseau, 12, déc. 2000. Dans la suite, les citations de Smith seront données dans l'une ou l'autre des traductions disponibles, ou traduites par moi-même, en mentionnant la référence dans l'édition anglaise de référence, puis dans la traduction choisie, notée $\mathrm{C}$ pour Théorie des sentiments moraux, Plan-de-la-Tour, Éd. d'Aujourd'hui, 1982, reprint de l'édition Guillaumin 1860, et BGP pour Théorie des sentiments moraux, Paris, Presses universitaires de France, 1999.

84. Theory of moral sentiments, I.i.4.6-7, in SмITH, vol. I, p. 21 (BGP, p. 45).

85. Ibid., I.i.1.3, in SMITH, vol. I, p. 10 (C, p. 2-3).

86. Cité in D. D. Raphael et A. L. MacFie, introd. à Theory of moral sentiments, in SMith, vol. I, p. 3. 
Malgré ses protestations de modestie ${ }^{87}$, Smith n'a besoin de personne pour faire cette comparaison. Dans un essai antérieur à la Théorie, il annonçait son ambition: dans le système de Newton, une fois admis le principe de l'universalité de la gravitation, tous les phénomènes expliqués suivent nécessairement, et leur connexion n'est pas «générale et relâchée (loose), comme dans la plupart des autres systèmes », mais « partout la plus précise et détaillée que l'on puisse imaginer ${ }^{88}$. C'est aussi ce qu'il répète depuis dix ans dans ses cours de rhétorique : dans les écrits didactiques, la méthode newtonienne de composition donne un «plaisir [...] bien supérieur à celui que l'on tire de la méthode déliée (unconnected) où chaque chose est expliquée pour elle-même sans référence aux autres ${ }^{89}{ }^{\prime}$. Lecteur attentif de la Théorie des sentiments moraux, Burke ne dira pas autre chose.

La méthode newtonienne est-elle praticable en morale? Smith y parvient-il vraiment? Toute jalousie mise à part (mais ce n'est pas si simple), la Théorie, qui paraît si bien accomplir son ambition, soulève tout de même quelques objections. La plus importante vient de Hume lui-même, au détour d'un reproche qui en dit long («[...] vous ne devriez pas être si absorbé par votre livre au point de ne jamais mentionner le mien $\gg)^{90}$ :

«Je regrette que vous n'ayez pas apporté la preuve détaillée que toutes les sortes de sympathie sont nécessairement agréables. [...] Il est évident qu'un homme de mauvaise humeur, fatigué et dégoûté de tout, toujours ennuyé [en français dans le texte], un homme maladif et qui se plaint, jette un froid sur la compagnie. Ce qui s'explique, je suppose, par la sympathie. Mais alors cette sympathie est désagréable. »

La vue d'un homme qui se plaint et qui souffre, ajoute Hume, est désagréable parce que la sympathie nous fait partager son sentiment, son état: comment Smith peut-il alors prétendre, contre l'évidence, que tout effet de sympathie est agréable? D'autant qu'il semble soutenir ailleurs une idée contraire à la première : "Vous dites expressément qu'il est pénible de partager la douleur et que cela se fait toujours à regret. » Voilà qui nécessiterait, au sens fort du terme, une explication : «Il me semble que vous devriez modifier ou expliquer ce sentiment, et le réconcilier avec votre système. » Avec sa tranquille assurance, la réponse de Smith dut être déroutante : sans changer une ligne à son texte, il se contente d'une note en bas de page dans la seconde édition (1761). Pour éviter toute confusion, dit-il,

87. J. C. BRyce, introd. à Lectures on rhetoric and belles-lettres, in SMITH, vol. IV, p. 24.

88. History of astronomy, in Sмiтн, vol. III, p. 104.

89. Lectures on rhetoric and belles-lettres, in SMITH, vol. IV, p. 146.

90. Hume vient de publier son History of England. Les extraits de Hume sont tirés de sa lettre du 28 juillet 1759, in The Correspondence of Adam Smith, in SMith, vol. VI, p. 43. 
il faut bien distinguer entre : $1^{\circ}$ le sentiment sympathique proprement dit, qui «peut être agréable ou désagréable suivant la nature de l'affection originelle qui l'a fait naître », puisqu'il en conserve "toujours, à quelques égards », le caractère; $2^{\circ}$ un autre sentiment, toujours agréable, voire délicieux, qui se forme dans l'âme du spectateur en «observant la parfaite coïncidence [...] entre l'affection sympathique qu'il éprouve et l'affection originelle de la personne intéressée ${ }^{91}$, et qu'on appelle le «plaisir de la sympathie réciproque ${ }^{92} »$. Pour Smith, tout est clair, et l'incident est clos : « Je crois que je lui ai infligé une défaite totale (I think I have entirely discomfitted him), confie-t-il au moment de donner sa réponse ${ }^{93}$.

Dans l'état le plus avancé de la recherche aujourd'hui, nous ressemblons trop souvent à Hume : nous ne comprenons pas ce petit quelque chose qui donne aux yeux de Smith tout son sens à sa Théorie. Parce que nous peinons à lire le livre aussi bien que Burke : tout simplement dans l'ordre des chapitres et des parties, comme le voulait Smith; et surtout dans les deux premières éditions (1759 et 1761) plutôt que dans la sixième (1790), qui éloigne le livre de son projet initial ${ }^{94}$. Pour respecter la chaîne des raisonnements liés entre eux, depuis le premier des principes (la sympathie) jusqu'au dernier des phénomènes (la variation des "caractères » nationaux). On cherchera donc d'abord dans la lecture la cohérence d'un système : un système où les métaphores de la physique et de l'optique se recoupent et rivalisent d'efficacité (comme ces deux sciences chez Newton). Mais cette lecture ne sera possible que si l'on restaure, dans le même temps, le contexte concret qui donne un sens autre que logique à ce système : non pas seulement comment Smith raisonne, mais, en premier lieu, de quoi il parle.

La sympathie envers la joie et la peine : c'est la question qui sépare Hume et Smith, et donne à celui-ci son air de supériorité. Plus que jamais maître de rhétorique, Smith prend bien soin d'exposer en avant l'idée commune, l'observation évidente qui veut que «notre tendance à sympathiser avec la peine doit être très forte, et notre inclination à sympathiser avec la joie très faible ${ }^{95} »$, entre autres raisons parce que la peine est une sensation

91. Theory of moral sentiments, I.iii.1.9*, in Sмгтн, vol. I, p. 46 (C, p. 49).

92. Ibid., I.i.2, in SмIтн, vol. I, p. 13 (BGP, p. 32).

93. Lettre du $1^{\text {er }}$ octobre 1759, dans The Correspondence of Adam Smith, in SMITH, vol. VI, p. 49.

94. La nouvelle traduction de Biziou, Gautier et Pradeau reprend malheureusement ce principe (même si elle signale la plupart des modifications apportées lors de la sixième édition). Il manque une véritable édition/traduction qui présenterait séparément le texte de 1761 et les ajouts de 1790. Sur les objectifs spécifiques de Smith dans la sixième édition, voir SHinohara, 1993.

95. Theory of moral sentiments, I.iii.1.4, in SмIтH, vol. I, p. 44. On trouve cette idée chez Rousseau, p. ex., voir Émile, liv. IV, in Rousseau, 1959-1995, vol. IV, p. 504 : «L'imagination nous met à la place du misérable plutôt qu'à celle de l'homme heureux; on sent que l'un de ces états nous touche de plus près que l'autre. » 
plus vive que le plaisir, et par conséquent la sympathie pour la peine une «perception plus forte et plus distincte» que la sympathie pour la joie ${ }^{96}$. Mais il ajoute aussitôt, dans un retournement dramatique : "En dépit de ce préjugé, j'oserai cependant affirmer (I will venture to affirm) que notre inclination à sympathiser avec la joie, lorsqu'elle n'est contrariée par aucun sentiment d'envie, est plus forte que notre inclination à sympathiser avec la peine $^{97}$.» Comment justifier cette audace? Par une asymétrie naturelle, un fait d'expérience, qui situe l'état ordinaire de l'homme, c'est-à-dire le spectateur ordinaire, beaucoup plus près de la joie que de la peine :

«L'adversité [...] abat (depresses) l'âme de celui qui souffre beaucoup plus endessous de son état naturel que la prospérité ne peut l'élever au-dessus. Le spectateur aura donc beaucoup plus de difficulté à sympathiser entièrement [...] avec son chagrin que de partager sincèrement sa joie : il doit s'éloigner (depart) beaucoup plus de son propre état d'esprit naturel dans un cas que dans l'autre ${ }^{98}$.»

Selon un principe attribué aux stoïciens (Smith évoque bientôt la mort de Caton, dans le récit de Sénèque ${ }^{99}$ ), l'état naturel de bonheur, celui de l'« homme qui est en bonne santé, sans dette, et qui a une conscience claire », est pratiquement égal pour tous, puisque séparé du niveau le plus élevé de bonheur par un écart «infime » ${ }^{100}$. C'est donc là que se trouve le spectateur dans la Théorie : tout près de la joie. En se tournant maintenant de l'autre côté, vers la peine, on constate au contraire qu'entre l'état naturel du spectateur et le point le plus bas de la misère, « la distance est immense et prodigieuse ${ }^{101} »$. Nous sommes « conscients qu'il y a un intervalle bien plus grand dans un cas que dans l'autre ${ }^{102} »$. Ainsi cette différence de distance vient-elle compenser la différence inverse des intensités. La peine est une sensation plus vive, mais le spectateur ordinaire, étant beaucoup plus loin de l'homme dans la peine que de l'homme dans la joie, sera en fin de compte plus en sympathie avec la joie qu'avec la peine : «Notre gaieté à l'occasion d'un baptême ou d'un mariage vient toujours du cœur, sans aucune affectation», et «toutes les fois que nous félicitons cordialement nos amis [...], leur joie devient littéralement notre joie», mais «au contraire, quand nous exprimons nos condoléances à nos amis dans leurs afflictions, combien peu nous sentons, comparé à ce qu'ils sentent ${ }^{103}$ !

96. Theory of moral sentiments, I.iii.1.3, in Sмітн, vol. I, p. 44.

97. Ibid., I.iii.1.4-5, in SмITH, vol. I, p. 44-45.

98. Ibid., I.iii.1.8, in Sмiтн, vol. I, p. 45.

99. Ibid., I.iii.1.13, in SMiTH, vol. I, p. 48.

100. Ibid., I.iii.1.7-8, in SмITH, vol. I, p. 45 (BGP, p. 85-86).

101. Ibid., I.iii.1.8, in SMITH, vol. I, p. 45.

102. Ibid., I.iii.1.6, in SMITH, vol. I, p. 45.

103. Ibid., I.iii.1.11-12, in SмIтн, vol. I, p. 47 (BGP, p. 87). 
La relation agent/objet/spectateur est donc inscrite dans un espace, dans un jeu de distances, où les métaphores de la vision et du déplacement jouent le premier rôle ${ }^{104}$. Mais de quel espace s'agit-il ? D'un espace purement mental, imaginaire ou, au contraire, d'un espace qui conserve une dimension physique, sensible? Il faut insister ici à nouveau sur l'écart entre Smith et Hume. Confondant espace physique (géographique) et espace imaginaire (des relations), Hume développe dans son Traité de la nature humaine (1739) la métaphore courante de la distance affective (sans y penser, nous nous disons plus proches de nos amis et plus éloignés de simples relations) : «[...] les hommes sont en grande part gouvernés par l'intérêt », leur préoccupation ne va jamais « très loin $[\ldots]$ au-delà d'eux-mêmes », si bien que «dans la vie courante, il ne leur est pas habituel de regarder plus loin que leurs amis et leurs relations les plus proches ». Comme nous éprouvons davantage de sympathie pour nos voisins que pour nos compatriotes, pour nos compatriotes que pour des étrangers, nous avons besoin d'une éducation morale qui vienne s'opposer à cette tendance, qui nous apprenne la raison et sa conduite impartiale, « fondée sur quelque vue lointaine et réflexion distante ${ }^{105} \gg$.

Au contraire, en développant, à partir de An essay towards a new theory of vision de George Berkeley (1709), une autre métaphore, celle de l' « œil de l'esprit » (eye of the mind), Smith situe la relation entre agent et spectateur dans un espace entièrement imaginaire. L'œil de l'esprit analogue à l'œil du corps, cela veut dire qu'à l'intérieur de notre conscience certains objets sont plus ou moins faciles à penser, comme s'ils étaient plus ou moins éloignés, donc plus ou moins faciles à voir : par exemple, l'idée d'une prison nous déplaît, parce que l'effet immédiat de la prison (le « confinement des malheureux qui y sont enfermés ») est désagréable, alors que ses «effets éloignés », qui sont utiles à la société, ne sont pas pris en compte, car «l'imagination ne prend pas le temps d'aller jusqu'à se [les] représenter » ou «les voit à une trop grande distance pour en être très affectée ${ }^{106}$. Mais cet effet d'éloignement ne concerne pas la plus ou moins grande familiarité entre agent et spectateur. La sympathie de Smith se veut universelle, sans distinction d'amitié ou de relation, parce que sans distinction d'intérêt. Comme la conversation en société, ou la conversation

104. Il faut souligner que Smith, qui a critiqué le style métaphorique dans ses cours de rhétorique, atténue fréquemment ses métaphores en employant les expressions consacrées «as it were », « if I may say so », équivalentes du «pour ainsi dire » recommandé par Du Marsais dans son Traité des tropes, voir Encyclopédie, article « Métaphore ».

105. Hume, 1993, liv. III 2 7, p. 143 et liv. III 3 1, p. 205.

106. Theory of moral sentiments, I.ii.3.4, in SMITH, vol. I, p. 35 (BGP, p. 69). Sur l'influence de Berkeley sur la théorie optique de Smith, énoncée dans Of the external senses, in SMITH, vol. III, voir Ross, 1995, p. 76-77 et 412. 
de $c l u b$, son modèle implicite, elle dessine un espace de relations abstraites entre inconnus, mis à égalité pour un temps, le temps du loisir ${ }^{107}$.

Bien sûr, on pourra reprocher à Smith d'exclure de son système rien moins que... les femmes, les larmes, les sauvages, les courtisans français, les Grands, les Italiens, les miséreux, les sages, les solitaires, les mondains, les opinions sur les sciences et les arts, les passions du corps, l'amour! La sympathie ne s'applique en réalité à aucun de ces cas de figure ${ }^{108}$ ! La Théorie n'est donc pas universelle, comme elle le prétend, même si elle s'appuie sur une sorte d'homme universel (de même que l'Émile de Rousseau met en scène un «homme abstrait», représentant «la condition humaine ${ }^{109}$ ) : un « homme privé » ou homme ordinaire, sain d'esprit, etc. ${ }^{110}$. Une figure, un idéal, quelque chose comme l'aurea mediocritas d'Horace (l'heureuse médiocrité), pour autant que Smith le croit incarné dans son public et en lui-même (dans la limite de sa propre tendance à la mélancolie) : un idéal à l'opposé de l'extravagance d'un Samuel Johnson, son ennemi intime, capable de tomber en prière au milieu d'un dîner en ville pour se faire remarquer ${ }^{111}$. Mais le propos de Smith n'est pas désincarné pour autant. Il parle de reconnaissance, de bonté, de jalousie, de colère et de toutes sortes de sentiments bien réels. Il donne des exemples, brosse des portraits dignes d'un traité des caractères : le parvenu, le petitmaître (coxcomb), l'officier, le brigand, etc. Il fait varier les rangs, les conditions, les classes, les âges, les coutumes (nationales), mais aussi les circonstances de la rencontre (à ne pas confondre avec les conditions sociales). Ce qu'il appelle «société » n'est donc pas un concept philo-

107. La Richesse des nations et la Théorie des sentiments moraux ne sont donc pas en contradiction, comme le pensaient les historiens allemands à la fin du XIX ${ }^{\mathrm{e}}$ siècle, puisqu'ils ne font que théoriser deux moments différents de la vie du bourgeois : le travail du jour et son principe d'intérêt; le loisir du soir (la conversation, le club, le cercle, etc.) et ses règles de convenance et de sympathie. L'interprétation dominante en France aujourd'hui considère l'intérêt comme une modalité de la sympathie, manière élégante mais inutile de résoudre le fameux «Problème Adam Smith ». Il faudrait plutôt dire que la sympathie est le loisir de l'intérêt. Sur la revendication bourgeoise d'un temps de loisir, qui n'est plus l' "amusement » des nobles, voir Mauzi, 1969, et Groethuysen, 1977. Sur la question en Grande-Bretagne, voir LANGFORD, 1989.

108. Comme le soulignent Biziou, Gautier et Pradeau, Smith écrit pour un public masculin : à la différence de l'homme, la femme fait preuve naturellement d' « humanité », sentiment qui ne demande « aucune abnégation, aucune maîtrise de soi, aucun usage remarquable du sens de la convenance », et qui ne rentre donc pas dans le système de la Théorie, voir Theory of moral sentiments, IV.2.10, in SмITH, vol. I, p. 191 (BGP, p. 265). De la même manière, le sauvage, vivant «dans un danger continuel », " ne compte sur aucune sympathie », « de quelque nature que soit sa douleur», voir ibid., V.2.9, in Smith, vol. I, p. 205 (C, p. 238). De la même manière, Smith donne une raison logique pour chaque catégorie qu'il exclut de son système.

109. Émile, liv. I, in Rousseau, 1959-1995, vol. IV, p. 252.

110. Theory of moral sentiments, I.iii.2.5, in SмIтн, vol. I, p. 55.

111. Voir Ross, 1995, p. 335. Smith a fait la critique du Dictionnaire de la langue anglaise de Samuel Johnson dans l'Edinburgh Review, in SмIтH, vol. III, p. 232-233. 
sophique mais un monde bien réel, qui doit respecter, avant toute chose, la politesse et la civilité, comme si ses lois décalquaient les lois de la conversation, comme si la société civile était formée de compagnies ou d'assemblées de compagnons, dont la réunion reste problématique ${ }^{112}$. Ainsi, parce que «la moitié de l'humanité » manque de la « réserve nécessaire lorsque l'on parle de ses amis » ou « de ses études », « un philosophe ne fait bonne compagnie qu'à un autre philosophe, et le membre d'un club qu'à son petit noyau de compagnons ${ }^{113}$ ! À l'intérieur de la littérature plus ou moins fantaisiste des caractères et des coutumes, dans le vaste domaine des traités de civilité et de morale, Smith veut seulement parler de ce qu'il connaît à des auditeurs (et bientôt à des lecteurs) qu'il connaît : des hommes, des bourgeois, des Écossais, des membres de divers clubs (ces clubs qu'il a beaucoup fréquentés lui-même). De même que l'admirable système de Newton reposait en réalité sur le phénomène le plus familier (la gravité à la surface de la Terre), la Théorie des sentiments moraux se veut le plus «philosophique ${ }^{114} \gg$ des systèmes moraux, appuyé sur les réalités les plus familières pour un bourgeois de 1760 : la grande affaire des conditions, des sentiments et du bonheur, sanctionnée par l'impitoyable ridicule ${ }^{115}$. Sa matière est la matière des romans et du théâtre de son temps, sans être en avance sur lui (Smith est plus proche d'Oliver Goldsmith ou de Samuel Richardson que de Henry Fielding, plus proche de Marivaux et Crébillon — qu'il admire - que du drame bourgeois de Diderot) ${ }^{116}$. Son ambition reste profondément classique : là où Hume cherche à étudier la succession rapide

112. Theory of moral sentiments, III.5.2, in Sмітн, vol. I, p. 163; I.i.4.5, in Sмітн, vol. I, p. 21; I.ii.4.3, in Sмiтh, vol. I, p. 40. Les mots «compagnie» et «compagnon» sont employés à plusieurs reprises dans la partie I : Theory of moral sentiments, in SMITH, vol. I, p. 20, 23, 27, 34, 41, 42 et 47.

113. Theory of moral sentiments, I.ii.2.6, in $\mathrm{S}_{\mathrm{MITH}}$, vol. I, p. 34.

114. Voir History of astronomy, in SMITH, vol. III, p. 98 et 104; Lectures on rhetoric and belles-lettres, in Sмiтн, vol. IV, p. 146.

115. Dans les deux premières éditions, le véritable obstacle à la sympathie n'est pas l'envie, comme le suppose Jean-Pierre Dupuy, in Dupuy, 1992, mais le ridicule, avec tous ses attributs : le rire, la moquerie, la dérision, le mépris, l'humiliation. Sujet à la mode, élevé au rang de problème philosophique (l'Académie française en a fait son sujet de concours en 1753 : «Si la crainte du ridicule étouffe plus de talents et de vertus qu'elle ne corrige de vices et de défauts »), le ridicule est un passage obligé du cours de rhétorique de Smith, autour de Lucien de Samosate et de Jonathan Swift, qui à eux deux « ont formé un système complet du ridicule », c'est-à-dire un «système de morale dont on peut tirer des règles de vie plus saines et plus justes que de la plupart des systèmes de morale établis », voir Lectures on rhetoric and belles-lettres, in $\mathrm{S}_{\mathrm{MiTH}}$, vol. IV, p. 124 et 50-51. Sur l'importance du ridicule et le rôle marginal de l'envie, voir LeFEBvre, 1998a, p. 338-343 et 366-369.

116. En opposant Richardson à Fielding, on souligne généralement que l'idéologie dite «bourgeoise » des romans de Richardson reste en réalité conforme à une hiérarchie sociale inégalitaire et aristocratique dans son esprit, voir GunN, 1997. Dans la sixième édition, Smith conseillera de lire « les poètes et les romanciers qui ont le mieux peint l'amour, l'amitié, les affections privées et domestiques, comme Racine, Voltaire, Richardson, Marivaux et Riccoboni », voir Theory of moral sentiments, III.3.14, in Sмітн, vol. I, p. 143. Les écrits esthétiques de Smith sont maintenant bien connus en France, mais le lien avec la Théorie des senti- 
des passions dans l'esprit (et fonde la psychologie moderne de l'association), Smith s'appuie encore sur le vocabulaire classique des sentiments, considérés pour ce qui les distingue (par exemple : la bonté, le ressentiment et la joie n'ont pas les mêmes propriétés vis-à-vis de la sympathie) ${ }^{117}$.

C'est parce que l'esprit fonctionne comme la vision que l'on sympathise plus avec la joie, et «c'est parce que les hommes sont plus disposés à sympathiser complètement avec notre joie qu'avec nos chagrins, que nous faisons parade de nos richesses, et que nous dissimulons notre pauvreté ${ }^{118}$ », principe garant de l'ordre social. Ainsi s'articulent en système les trois chapitres de la première partie de la Théorie, elle-même fondation du livre: principe de convenance (propriety); catalogue des passions; distinction des rangs. Ce sont les variations d'échelle de la vision, définies par les lois de la perspective (la grandeur apparente d'un objet diminue en raison inverse de sa distance), qui définissent le personnage du spectateur, et son jumeau le «spectateur impartial», personnification de notre conscience morale, explicitement comparé à la position qu'il faut adopter pour voir plusieurs objets en perspective et rétablir les vraies grandeurs des choses :

«Comme, devant l'œil du corps, les objets apparaissent grands ou petits non pas selon leurs dimensions réelles, mais plutôt selon leur proximité ou leur distance, ils font de même devant ce qu'on peut appeler l'œil naturel de l'esprit : et l'on remédie aux défauts de ces deux organes exactement de la même manière. Dans la position où je me trouve actuellement, un paysage immense, couvert de bois, de montagnes lointaines, semble n'occuper que le petit espace de la fenêtre devant laquelle j'écris; il semble être hors de toute proportion avec la chambre où je suis. Le seul moyen que j'aie pour comparer les petits objets qui sont autour de moi et ces grands objets éloignés, est de me transporter, au moins par l'imagination, dans une autre position, d'où je pourrai tous les voir à peu près de la même distance et par conséquent juger leurs véritables proportions. L'habitude et l'expérience m'ont appris à le faire si facilement et si spontanément que je suis à peine conscient de le faire.

« De la même manière, suivant les passions égoïstes et originelles de la nature humaine, la perte ou le gain d'un tout petit intérêt à soi apparaît beaucoup plus important, excite une joie ou une peine beaucoup plus passionnée, un bien plus grand désir ou aversion, que le plus grand souci d'une autre personne avec qui nous n'avons pas de lien particulier. Les intérêts de cette personne, aussi longtemps qu'ils sont considérés depuis notre position, ne peuvent jamais être mis en balance avec le nôtre, ne peuvent jamais nous empêcher d'œuvrer par tous les moyens à la promotion du nôtre, aussi ruineux qu'il puisse être pour elle. Avant de pouvoir faire une bonne comparaison de ces intérêts opposés,

ments moraux n'est pas encore fait de manière systématique, voir ABOUDRAR, 1998, et CARBoni, 1997.

117. Sur Hume, voir CLÉRO, 1985.

118. Theory of moral sentiments, I.iii.2.1, in $\mathrm{S}_{\mathrm{MITH}}$, vol. I, p. 50 (C, p. 53-54). 
il nous faut changer de position. Nous ne devons les voir, ni de notre position, ni de celle de cette personne, ni avec nos yeux, ni avec les siens, mais de la position et avec les yeux d'une troisième personne, qui n'a pas de lien particulier avec aucun de nous deux et qui juge entre nous avec impartialité. Là encore, l'habitude et l'expérience nous ont appris à faire cela si facilement et si spontanément que nous sommes à peine conscients de le faire ${ }^{119}$. »

Ainsi la clôture du système, parfaitement voulue, donne à la sympathie un statut analogue à l'attraction chez Newton : premier principe, dont on ne connaît pas la nature, seulement les effets. L'essentiel est donc ailleurs, dans la comparaison parfaitement analogique de l'œil et de l'esprit. En refusant toute sympathie par voisinage physique (une des définitions traditionnelles du mot), en ne mettant en relation que des agents pratiquement inconnus les uns des autres, Smith refuse toute distance affective, toute relation de cause à effet entre l'œil et l'esprit, et peut ainsi prétendre à une véritable analogie, au sens du $\mathrm{XVIII}^{\mathrm{e}}$ siècle — jusque dans la reproduction des échelles de distance et des calculs d'optique propres à la perspective. Pourtant, le système semble incomplet: Smith insiste sur le fait que son système de sympathie ne nécessite aucune éducation raisonnée, qu'il fonctionne par apprentissage spontané de la convenance à donner aux sentiments dans la fréquentation des hommes, mais il reste imprécis sur ce phénomène. Tout comme le laisse perplexe, au fond, l'apprentissage par l'œil des règles de la perspective : est-ce « par un instinct particulier, ou bien par une certaine application de la raison ou de l'expérience ${ }^{120} »$ ? En se désintéressant de la question, Smith montre, une fois de plus, qu'il est un homme parfaitement cohérent ${ }^{121}$.

\section{POUR UNE HISTOIRE CONCRÈTE}

Par un curieux effet qu'on pourrait appeler, comme dans la littérature policière, excès d'évidence, ni Bauclair, ni Berthier, ni aucun contemporain de Rousseau ne reconnaissent la montre dans le Contrat social — pas plus apparemment qu'aucun lecteur ultérieur, même aussi attentif que Joseph de

119. Theory of moral sentiments, III.3.2-3, in SмIтH, vol. I, p. 135-136.

120. Of the external senses, in Sмiтн, vol. III, p. 151.

121. Toujours sûr de son fait, Smith signale lui-même - et justifie par la toute-puissance divine - le passage où son système présente encore une «irrégularité » (comme les systèmes des astronomes avant Newton), voir Theory of moral sentiments, II.iii.3, in SMITH, vol. I, p. 104-108. 
Maistre, ou, plus tard, Maurice Halbwachs ${ }^{122}$. À partir de la fin du $\mathrm{XVIII}^{\mathrm{e}}$ siècle, cela s'explique assez bien: la métaphore de la montre a changé de valeur, elle devient comme chez Jules Verne synonyme de perfection, de perfection inhumaine, comme les montres elles-mêmes ${ }^{123}$. Le mot est resté le même, mais la chose et l'idée ont basculé de valeur, du bien au mal. Nous ne savons donc plus vraiment ce que nous voulons dire lorsque nous déplorons les... «rouages de l'administration ». Est-ce que nous pensons encore à des roues dentées qui tournent les unes dans les autres à des vitesses différentes? Ou simplement à une grande machine indistincte dont nous sommes les captifs? Pour nous, c'est une image à tout faire. Pour Rousseau, cela n'aurait été, au contraire, qu'une «métaphore sans métaphore »: l'extension d'un sens propre, technique, à un autre domaine, où il pensait retrouver des effets comparables de temps, de force, de frottement, de réglage. Autrement dit: une analogie.

De la même manière, Smith avance suffisamment masqué pour ne pas laisser prise à la satire : sa « sympathie » a certains attributs de l'attraction newtonienne, mais il se garde bien de le proclamer. Son analogie de l'œil et de l'esprit est contrôlée jusqu'au bout, mais il n'en fait pas davantage de publicité. Il est bien trop prudent (et orgueilleux) pour cela. Tout dépend, alors, de la sagacité des lecteurs (voire des traducteurs, dans le cas de la réception française, par exemple). Kant est un cas intéressant : pseudonewtonien dans sa physique, partisan d'une mesure mathématique de l'esprit en plus ou moins (la fameuse équation des plaisirs et des peines : « $4 a-a=3 a »)$, il limite sa conception de ce qu'il appelle l' « insociable sociabilité » des hommes à deux sentiments (la vanité et l'envie) et ne s'intéresse qu' au passage du «point de vue » individuel au «point de vue » du tout, sans s'arrêter au système ternaire agent/objet/spectateur qui faisait la force de Smith. Il transforme donc la métaphore de la vision sociale en une vision impossible, inaccessible à l'homme, celle que l'on pourrait avoir en se plaçant au «point de vue » du soleil ${ }^{124}$. Ce n'est pas de ce côté qu'il faudrait chercher la postérité de la Théorie des sentiments moraux (pour autant qu'elle existe), et pas non plus chez Bentham ou chez Cabanis, qui trahissent également le projet de Smith, mais plutôt chez $\mathrm{M}^{\mathrm{me}}$ de Staël, en France, ou chez Darwin, en Angleterre ${ }^{125}$.

122. Voir Maistre, 1991, et Rousseau, 1943.

123. Sur le modèle de la montre chez Jules Verne, voir Chesneaux, 1971, p. 35-40, et RayMOND, 1974.

124. Voir KANT, 1980-1986, vol. I, p. 276; Idée d'une histoire universelle au point de vue cosmopolitique, cité in SIMON, 1997, p. 171; Le Conflit des facultés, cité in FERRY, 1986, p. 411. Pour une discussion du pseudo-newtonisme de Kant, voir Lefebvre, 1998a, p. 460467.

125. Sur le rapport entre sentiments et types de société chez Germaine de Staël, voir REDDy, 1997, p. 338-339. Sur Charles Darwin, admirateur du premier chapitre de la Théorie des sentiments moraux, voir TORT, 1996, p. 4035. 
Pourquoi s'interroger sur cette postérité ? Parce que les grandes théories sociologiques du XIX ${ }^{\mathrm{e}}$ siècle, de Quételet à Durkheim, en passant par Comte et par Spencer, ont hérité, parfois sans le savoir, parfois sans le vouloir, des comparaisons raisonnées familières au XVIII ${ }^{\mathrm{e}}$ siècle: impossible de comprendre le conflit de Comte et de Quételet, par exemple, ou de Tarde et de Durkheim, sans rapporter leurs mots et leurs métaphores aux choses du régulateur, du frottement, de l'équilibre, de la distance ou de l'attraction en ajoutant, bien sûr, les choses nouvelles, les choses de l'électricité, de la biologie, du magnétisme, bientôt du téléphone, etc. ${ }^{126}$. Des choses qui résistent à l'étude, qui font de vrais objets scientifiques ${ }^{127}$. Ainsi, lorsque nous employons des expressions comme « les rouages de l'administration » ou «le point de vue sociologique», nous ne faisons pas seulement un déplacement, un acte de métaphore (étymologiquement, un «transport»), mais aussi implicitement une proportion au sens du $\mathrm{XVIII}^{\mathrm{e}}$ siècle, une comparaison de chose à chose qui appelle la possibilité de la mesure. Comme dira plus tard Spencer, c'est une question d' 'échafaudage $^{128} »$ : les auteurs élégants (ou simplement le temps) enlèvent l'échafaudage quand l'œuvre est achevée. Mais l'image n'est pas si juste: en théorie sociale, enlever l'échafaudage, c'est prendre le risque de voir un jour le bâtiment tomber. En oubliant Newton et l'attraction - après la montre et la perspective -, nous nous interdisons de comprendre par quel échafaudage les théoriciens $\mathrm{du} \mathrm{xIX}^{\mathrm{e}}$ siècle ont bâti, comment cela les rapprochait bien davantage de leurs prédécesseurs qu'ils n'ont voulu le dire, et combien ils devraient être exotiques à nos yeux.

Frédéric LeFeBvRE (décembre 1999).

126. P. ex., sur le lien entre le régulateur de montre et le concept de régulation en biologie, voir CANGUilhem, 1977, p. 81-99.

127. Sur ce point, en référence à la définition de l'objet chez Durkheim, voir BRIAN, 1996.

128. L'expression est reprise par Durкнеiм, 1975, p. 374-375: « [...] ces métaphores et ces analogies avaient leurs avantages aux débuts de la science; suivant une expression de Spencer, ce sont d'utiles échafaudages, mais qui nous masquent la réalité. N'est-il pas temps de les jeter à bas pour nous mettre en face des choses? » 


\section{LISTE DES RÉFÉRENCES}

Encyclopédie = Encyclopédie ou Dictionnaire raisonné des sciences, des arts et des métiers, 1966-1995, $1^{\text {re }}$ éd. 1751-1772, ici réimpr., Stuttgart-Bad Cannstatt, Friedrich Fromman Verlag, 35 vol.

Nouveau dictionnaire de l'Académie françoise, 1994, $1^{\mathrm{re}}$ éd. 1718, ici Genève, Slatkine Reprints, 2 vol.

Aboudrar (Bruno-Nassim), 1998, «L'esthétique d'un économiste », Critique, 617, oct., p. 603-616.

Auroux (Sylvain), 1979, La Sémiotique des Encyclopédistes, Paris, Payot.

Bauclair (Paul Louis de), 1981, Anti-Contrat social dans lequel on réfute d'une manière claire, utile et agréable les principes posés dans le "Contrat social » de Jean-Jacques Rousseau, $1^{\text {re }}$ éd. 1765, ici Paris, Vrin.

Berthier (Guillaume François), 1988, Observations sur le "Contrat social » de Jean-Jacques Rousseau, $1^{\text {re }}$ éd. 1762, ici Reims, À l'Écart.

Boorstin (Daniel), 1983, Les Découvreurs, Paris, Robert Laffont (Bouquins).

Bourguinat (Élisabeth), 1998, Le Siècle du persiflage (1734-1789), Paris, Presses universitaires de France.

Bouveresse (Jacques), 1999, Prodiges et vertiges de l'analogie, Paris, Raisons d'agir.

Brian (Eric), 1994, La Mesure de l'État. Administrateurs et géomètres au XVIII 'eiècle, Paris, Albin Michel (L'Évolution de l'humanité).

Brian (É.), 1996, « Calepin. Repérage en vue d'une histoire réflexive de l'objectivation », Enquête, 2, p. 193-222.

Brown (Richard), 1989, Clefs pour une poétique de la sociologie, Le Méjan, Arles, Actes Sud.

BRUNET (Pierre), 1929, Maupertuis. L'œuvre et sa place dans la pensée scientifique et philosophique du XVIII siècle, Paris, Albert Blanchard, 2 vol.

Buffon (Georges Louis Leclerc, comte de), 1986, De la manière d'étudier et de traiter l'histoire naturelle, $1^{\text {re }}$ éd. 1749, ici Paris, Société des amis de la Bibliothèque nationale.

CANGuilhEm (Georges), 1977, Idéologie et rationalité dans l'histoire des sciences de la vie, Paris, Vrin.

CARboni (Pierre), 1997, «Les penseurs écossais des belles-lettres », in Pierre MorÈre, dir., Écosse des Lumières. Le XVIII ${ }^{e}$ siècle autrement, Grenoble, Éditions littéraires et linguistisques de l'université de Grenoble, p. 23-56.

CAssirer (Ernst), 1966, La Philosophie des Lumières, Paris, Fayard.

CHAPIro (Adolphe), 1991, La Montre française, du Xvi siècle jusqu'à 1900, Paris, L'Amateur.

Chesneaux (Jean), 1971, Une lecture politique de Jules Verne, Paris, Maspero.

Cléro (Jean-Pierre), 1985, La Philosophie des passions chez David Hume, Paris, Klincksieck. 
Condillac (Étienne Bonnot, abbé de), 1947-1951, Euvres philosophiques, Paris, Presses universitaires de France, 3 vol.

Coster (Michel de), 1978, L'Analogie en sciences humaines, Paris, Presses universitaires de France.

Duflo (Colas), 1998, «Le lien et la ficelle. Diderot, le lien social et les pantins », Recherches sur Diderot et l'Encyclopédie, 25, oct., p. 75-89.

Dupuy (Jean-Pierre), 1992, Le Sacrifice et l'envie. Le libéralisme aux prises avec la justice sociale, Paris, Calmann-Lévy.

Durkheim (Émile), 1925, L'Éducation morale, Paris, Presses universitaires de France.

Durkheim (É.), 1950, Leçons de sociologie. Physique des mœurs et du droit, Paris, Presses universitaires de France.

Durkheim (É.), 1967, De la division du travail social, $1^{\text {re }}$ éd. 1893, ici Paris, Presses universitaires de France.

Durkheim (É.), 1975, « Organisation et vie du corps social selon Schaeffle », in Textes. T. I : Éléments d'une théorie sociale, Paris, Minuit.

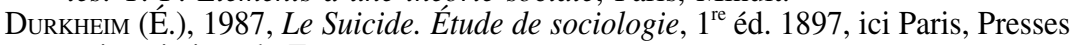
universitaires de France.

Ferry (Luc), 1986, « Kant », in François Châtelet, Olivier Duhamel et Évelyne PISIER, dir., Dictionnaire des æuvres politiques, Paris, Presses universitaires de France, p. 401-411.

GogGi (Gianluigi), 1987, «Polyphème mangeant les compagnons d'Ulysse. Une image politique de Diderot », Dix-Huitième siècle, 19, p. 339-346.

Gounier (Henri), 1970, La Jeunesse d'Auguste Comte et la formation du positivisme, $1^{\text {re }}$ éd. 1933, ici Paris, Vrin, 3 vol.

Goulemot (Jean-Marie), 1989, La Littérature des Lumières en toutes lettres, Paris, Bordas.

Groethuysen (Bernard), 1977, Origines de l'esprit bourgeois en France. Vol. I : L'Église et la bourgeoisie, Paris, Gallimard (Tel).

GunN (Daniel P.), 1997, « Is Clarissa bourgeois art? », Eighteenth-Century Fiction, vol. X, 1, oct., p. 1-14.

Gusdorf (Georges), 1971, Les Principes de la pensée au siècle des Lumières, Paris, Payot.

Gusdorf (G.), 1973, L'Avènement des sciences humaines au siècle des Lumières, Paris, Payot.

Hamou (Philippe), éd., 1995, La Vision perspective (1435/1740). L'art et la science du regard, de la Renaissance à l'âge classique, Paris, Payot.

Hirschman (Albert O.), 1980, Les Passions et les intérêts, Paris, Presses universitaires de France.

Hume (David), 1973-1974, « Pourquoi écrire des essais?», in Les Essais esthétiques, Paris, Vrin, 2 vol.

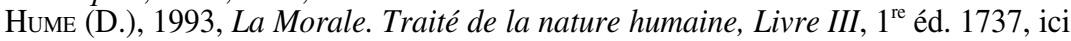
éd. Philippe Saltel, Paris, Flammarion (GF).

Jost (François), 1961, Jean-Jacques Rousseau suisse. Étude sur sa personnalité et sa pensée, Fribourg, Éditions universitaires, 2 vol.

Kant (Emmanuel), 1980-1986, Euvres philosophiques, Paris, Gallimard (Bibliothèque de la Pléiade), 3 vol.

LABUSSIËRE (Jean-Louis), 1994, «Puissance et impuissance de la mesure au siècle des Lumières », in Jean-Claude BEAune, dir., La Mesure. Instruments et philosophies, Seyssel, Champ Vallon, p. 244-251.

LANGFORD (Paul), 1989, A polite and commercial people. England 1727-1783, Oxford, Clarendon Press (The New Oxford History of England). 
Lefebvre (Frédéric), 1998a, La Montre et l'univers. Métaphores et modèles de la société (de Jean-Jacques Rousseau à Émile Durkheim), thèse de doctorat, Paris, université Paris VII-Denis Diderot.

Lefebvre (F.), 1998b, «Jean-Jacques Rousseau horloger malgré lui », La Revue, musée des Arts et Métiers, 24, sept., p. 34-41.

Le Men (Ségolène), dir., 1995, Lanternes magiques, tableaux transparents, Paris, Réunion des musées nationaux (Les Dossiers du musée d'Orsay, 57).

LÉvi-STrauss (Claude), 1993, Regarder, écouter, lire, Paris, Plon.

Loraux (Nicole), 1999, «La métaphore sans métaphore. À propos de l'Orestie », Europe, 837-838, janv.-fév., p. 242-264.

Loty (Laurent), 1995, La Genèse de l'optimisme et du pessimisme (de Pierre Bayle à la Révolution française), thèse de doctorat, Tours, université FrançoisRabelais.

MaGnin (Charles), 1981, Histoire des marionnettes en Europe, depuis l'Antiquité jusqu'à nos jours, $1^{\text {re }}$ éd. 1862, ici Genève, Paris, Slatkine.

MaISTRE (Joseph de), 1991, De la souveraineté du peuple. Un anti-Contrat social, $1^{\text {re }}$ éd. 1795, ici Paris, Presses universitaires de France.

Mandeville (Bernard), 1990, La Fable des abeilles, $1^{\text {re }}$ éd. 1714, Paris, Vrin, 2 vol.

MAuZI (Robert), 1969, L'Idée du bonheur dans la littérature et la pensée françaises au XVIII siècle, Paris, Armand Colin.

MeInoz (Jean), 1996, « Le droit de "mal écrire". Trois cas helvétiques $\left(\mathrm{XVIII}^{\mathrm{e}}-\mathrm{XIX} \mathrm{e}^{\mathrm{e}}\right)$ », Actes de la recherche en sciences sociales, 111-112, mars, p. 92-109.

Morelly, 1950, Code de la nature, $1^{\text {re }}$ éd. 1755, ici introd. et notes de Gilbert Chinard, Paris, Raymond Clavreuil.

Mumford (Lewis), 1973-1974, Le Mythe de la machine, Paris, Fayard, 2 vol.

NAMER (Gérard), 1979, Le Système social de Rousseau. De l'inégalité économique à l'inégalité politique, Paris, Anthropos.

Negri (Antonio), 1992, "La logique du temps et l'indécision du "Prince" », Rue Descartes, 3, janv., p. 97-112.

Perrot (Jean-Claude), 1992, Une histoire intellectuelle de l'économie politique, ${ }_{X V I I}{ }^{e}$-XVIII ${ }^{e}$ siècle, Paris, École des hautes études en sciences sociales.

Pintard (René), 1964, «L'humour de Rousseau », in Jean-Jacques Rousseau et son œuvre, actes du colloque de Paris, 1962, Paris, Klincksieck, p. 113-122.

Ratcliff (Marc James), 1997, "Le concept d'intensité dans la psychologie de Charles Bonnet », Revue d'histoire des sciences, vol. L, 4, p. 421-446.

RAymond (François), 1974, "L'homme et l'horloge », in Pierre André ToutTain, dir., Jules Verne, Paris, L'Herne, p. 141-151.

REDDY (William M.), 1997, «Against constructionism. The historical ethnography of emotions », Current anthropology, vol. XVIII, 3, June, p. 327-351.

Rosenblatt (Helena), 1997, Rousseau and Geneva. From the "First discourse" to the "Social contract", 1749-1762, Cambridge, Cambridge University Press.

Ross (Ian Simpson), 1995, The Life of Adam Smith, Oxford, Oxford University Press.

Rousseau (Jean-Jacques), 1943, Du contrat social, $1^{\text {re }}$ éd. 1762, ici comment. et notes par Maurice Halbwachs, Paris, Aubier Montaigne.

Rousseau (J.-J.), 1959-1995, Euvres complètes, Paris, Gallimard (Bibliothèque de la Pléiade), 5 vol.

SAvioz (Raymond), 1948, La Philosophie de Charles Bonnet de Genève, Paris, Vrin.

Schlanger (Judith), 1971, Les Métaphores de l'organisme, Paris, Vrin.

ShinohaRa (Hisashi), 1993, «The practical system of morality in Adam Smith », in Hiroshi Mizuta et Chuhei Sugiyama, dir., Adam Smith. International perspectives, New York, St. Martin's Press, p. 27-42. 
Simon (Pierre-Jean), 1997, Histoire de la sociologie, $1^{\text {re }}$ éd. 1991, ici $2^{\mathrm{e}}$ éd. Paris, Presses universitaires de France.

Sмiтh (Adam), 1976-1983, The Works and correspondence of Adam Smith, Oxford, Clarendon Press, 6 vol.

Starobinski (Jean), 1999, Action et réaction. Vie et aventures d'un couple, Paris, Seuil.

SteuART (James), 1967, An inquiry into the principles of political reconomy. Being an essay on the science of Domestic Policy in Free Nations, $1^{\text {re }}$ éd. 1767, in The Works political, metaphisical \& chronological of sir James Steuart, $1^{\mathrm{re}}$ éd. 1805, ici New York, Augustus M. Kelley, reprints of Economic classics, 6 vol.

TоRт (Patrick), 1989, La Raison classificatoire. Quinze études : les complexes discursifs, Paris, Aubier Montaigne.

ToRt (P.), 1996, «Smith Adam», in ID., dir., Dictionnaire du darwinisme et de l'évolution, Paris, Presses universitaires de France, 3 vol.

Touchefeu (Yves), 1995, « Rousseau et Homère », Dix-Huitième siècle, 27, p. 179190.

Tuzet (Hélène), 1988, Le Cosmos et l'imagination, Paris, José Corti. 